\title{
Nonperturbative structure of the ghost-gluon kernel
}

\author{
A. C. Aguilar, ${ }^{1}$ M. N. Ferreira, ${ }^{1,2}$ C. T. Figueiredo,,${ }^{1,2}$ and J. Papavassiliou ${ }^{2}$ \\ ${ }^{1}$ University of Campinas-UNICAMP, Institute of Physics "Gleb Wataghin," \\ 13083-859 Campinas, São Paulo, Brazil \\ ${ }^{2}$ Department of Theoretical Physics and IFIC, University of Valencia and CSIC, E-46100, Valencia, Spain
}

(Received 28 November 2018; revised manuscript received 25 January 2019; published 28 February 2019)

\begin{abstract}
The ghost-gluon scattering kernel is a special correlation function that is intimately connected with two fundamental vertices of the gauge sector of QCD: the ghost-gluon vertex, which may be obtained from it through suitable contraction, and the three-gluon vertex, whose Slavnov-Taylor identity contains that kernel as one of its main ingredients. In this work we present a detailed nonperturbative study of the five form factors comprising it, using as the starting point the "one-loop dressed" approximation of the dynamical equations governing their evolution. The analysis is carried out for arbitrary Euclidean momenta and makes extensive use of the gluon propagator and the ghost dressing function, whose infrared behavior has been firmly established from a multitude of continuum studies and large-volume lattice simulations. In addition, special Ansätze are employed for the vertices entering in the relevant equations, and their impact on the results is scrutinized in detail. Quite interestingly, the veracity of the approximations employed may be quantitatively tested by appealing to an exact relation, which fixes the value of a special combination of the form factors under construction. The results obtained furnish the two form factors of the ghostgluon vertex for arbitrary momenta and, more importantly, pave the way toward the nonperturbative generalization of the Ball-Chiu construction for the longitudinal part of the three-gluon vertex.
\end{abstract}

DOI: 10.1103/PhysRevD.99.034026

\section{INTRODUCTION}

The nonperturbative behavior of fundamental Green's functions of QCD, such as propagators and vertices, has received considerable attention in recent years [1-79] and is believed to be essential for acquiring a deeper understanding of the strong interactions. In this particular quest, the combined efforts between various continuum approaches $[1,6,7,9,27,46]$ and large-volume lattice simulations [51-61] have furnished a firm control on the infrared structure of the two-point sector of the theory (gluon, ghost, and quark propagators).

The case of the three-point functions (vertices) represents currently a major challenge, because, while their knowledge is considered to be crucial for both theory and phenomenology, their first-principle determination by means of conventional approaches is technically rather involved. In particular, such vertices possess, in general, rich tensorial structures, and their form factors contain three independent momenta. In order to determine the momentum dependence of vertex form factors, one may

Published by the American Physical Society under the terms of the Creative Commons Attribution 4.0 International license. Further distribution of this work must maintain attribution to the author(s) and the published article's title, journal citation, and DOI. Funded by SCOAP ${ }^{3}$. perform lattice simulations [62-70] or resort to continuum methods such as Schwinger-Dyson equations (SDEs) $[13,30,32,33,49,71,72,74-76]$ or the functional renormalization group [77-79]. Within these latter formalisms, the dynamical equations governing the momentum evolution of the vertices are derived and solved, under a variety of simplifying assumptions that reduce the inherent complexity of these calculations.

In a series of recent works $[25,31,39,41,80,81]$, the aforementioned approaches have been complemented by an alternative procedure, which exploits the Slavnov-Taylor identities (STIs) satisfied by a given vertex, and constitutes a modern version of the so-called "gauge technique" [82-85]. The main upshot of this method is to determine the nontransverse part of the vertex, ${ }^{1}$ in terms of the quantities that enter in the STIs, such as two-point functions and the so-called "ghost scattering kernels." These kernels correspond to the Fourier transforms of composite operators, where a ghost field and a quark or a gluon are defined at the same spacetime point. In the case of the quark-gluon vertex considered in the recent literature, the quantity in question is the "ghost-quark" kernel; its form factors have been reconstructed from the corresponding SDE in $[31,41]$,

\footnotetext{
${ }^{1}$ This part is usually referred to as "longitudinal" or "gauge" or "STI saturating."
} 


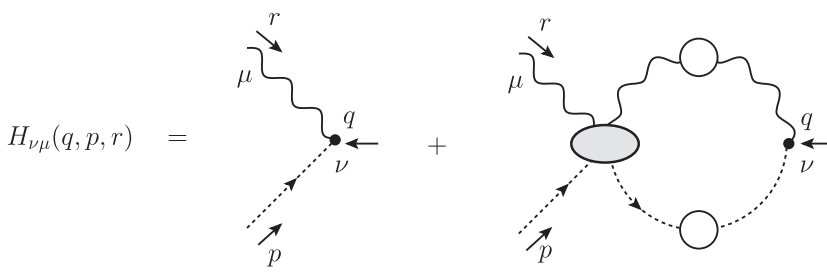

FIG. 1. The diagrammatic representation of the ghost-gluon scattering kernel. The tree-level contribution is given by $g_{\mu \nu}$.

and certain special kinematic configurations have been computed in $[25,80]$.

In the present work we turn our attention to the ghost-gluon kernel, to be denoted by $H_{\nu \mu}^{a b c}(q, p, r)=$ $-g f^{a b c} H_{\nu \mu}(q, p, r)$. The main objective is to compute from an appropriate SDE (see Fig. 1) the five form factors comprising this quantity, to be denoted by $A_{i}(q, p, r)$ $(i=1, \ldots, 5)$, for arbitrary Euclidean values of the momenta.

The interest in $H_{\nu \mu}$ and its form factors is mainly related with the two fundamental Yang-Mills vertices shown in Fig. 2 [86]. First, as was shown in the classic work of Ball and Chiu (BC) [87], the "longitudinal" part of the threegluon vertex, $\boldsymbol{\Gamma}_{\alpha \mu \nu}(q, r, p)$, may be fully reconstructed from the set of STIs that it satisfies [see Eq. (2.5)]. The ingredients entering in the $\mathrm{BC}$ "solution" are the gluon propagator, the ghost dressing function, and three of the form factors of $H_{\nu \mu}(q, p, r)$. Thus, in order to obtain reliable information on the infrared behavior of $\boldsymbol{\Gamma}_{\alpha \mu \nu}(q, r, p)$ by means of this method, the nonperturbative structure of the ghost-gluon kernel must be firmly established. Second, by virtue of an exact relation [see Eq. (2.4)], the ghost-gluon vertex, $\Gamma_{\mu}(q, p, r)$, which constitutes an important ingredient for a variety of SDE studies, is completely determined from the contraction of $H_{\nu \mu}(q, p, r)$ by $q^{\nu}$. Thus, knowledge of the $A_{i}(q, p, r)$ furnishes both form factors of $\Gamma_{\mu}(q, p, r)$ [88].

The methodology used for the computation of the $A_{i}(q, p, r)$ may be described as follows. The diagrammatic definition of $H_{\nu \mu}(q, p, r)$ shown in Fig. 1 involves the connected kernel $A^{\mu} A^{\rho} \bar{c} c$ (grey ellipse), whose skeleton expansion will be approximated by the "one-loop dressed" diagrams, depicted in Fig. 3; the basic quantities entering at this level are the gluon and ghost propagators, and the fully dressed vertices $\boldsymbol{\Gamma}_{\alpha \mu \nu}(q, r, p)$ and $\Gamma_{\mu}$. The individual form factors of $H_{\nu \mu}$ may then be isolated from the resulting equations by means of an appropriate set of projection operators. In the final numerical treatment we use the results of large-volume lattice simulations as input for the propagators, while for the vertices we resort to certain simplified Ansätze.

We next list the main highlights of our analysis: (i) we determine the form factors $A_{i}$ for general values of the Euclidean momenta, presenting the results in threedimensional (3D) plots, where $q^{2}$ and $p^{2}$ will be varied, for fixed values of the angle $\theta$ between them; (ii) the nonperturbative results obtained for $A_{i}$ are compared with their one-loop counterparts in three special kinematic limits; (iii) with the help of a constraint imposed by the STI [see Eqs. (2.13) and (2.14)], we quantify the accuracy and veracity of our truncation scheme; (iv) as a direct application, the various $A_{i}$ are fed into the Euclidean version of Eq. (2.8), giving rise to both form factors of the ghost-gluon vertex, for arbitrary momenta.

The article is organized as follows. In Sec. II we introduce the notation and set up the relevant theoretical framework. In Sec. III, we discuss the truncation scheme employed, and we define the set of projectors necessary for the derivation of the dynamical equations governing the form factors $A_{i}$. In Sec. IV we present the inputs and the additional approximations necessary for the numerical calculation of the $A_{i}$. Then, in Sec. V we present the numerical solution of the $A_{i}$ for general Euclidean momenta and compare them with the one-loop results for some special kinematic limits. Next, in Sec. VI we discuss how the constraint imposed by the STI may help us optimize the quality of the inputs used for the computation of the $A_{i}$. In Sec. VII we construct the two form factors of the ghostgluon vertex, carry out a comparison with the results of various approaches in the literature, and study their impact on the SDE of the ghost propagator. In Sec. VIII we present our discussion and conclusions. Finally, in Appendixes A and $\mathrm{B}$ we present the one-loop results for the $A_{i}$ in some special kinematic limits, for both "massive" and massless gluons, and certain lengthy expressions appearing in the derivation of the $A_{i}$.

\section{THEORETICAL BACKGROUND}

In this section we introduce the basic concepts and ingredients necessary for the study of $H_{\nu \mu}$ and elucidate on its connection with the ghost-gluon and three-gluon vertices. In addition, we introduce a particular relation, which is a direct consequence of the STI that $H_{\nu \mu}$ satisfies [87,89] and provides a nontrivial constraint on a combination of its form factors. We emphasize that throughout this article we work in the Landau gauge, where the gluon propagator $\Delta_{\mu \nu}^{a b}(q)=\delta^{a b} \Delta_{\mu \nu}(q)$ assumes the completely transverse form,

$\Delta_{\mu \nu}(q)=-i \Delta(q) P_{\mu \nu}(q), \quad P_{\mu \nu}(q)=g^{\mu \nu}-\frac{q^{\mu} q^{\nu}}{q^{2}}$.

In the case of a gluon propagator that saturates to a nonvanishing value in the deep infrared, it is natural to set (Euclidean space) $[90,91]^{2}$

\footnotetext{
${ }^{2}$ Contrary to the case of the quark propagator, this decomposition is not mathematically unique; however, the relevant dynamical equations severely restrict the possible structures [92].
} 


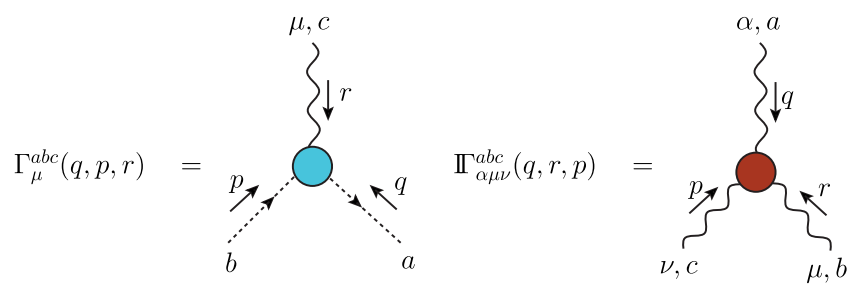

FIG. 2. Diagrammatic representations of the ghost-gluon (left) and three-gluon (right) vertices, and the adopted convention for their momenta dependence.

$$
\Delta^{-1}(q)=q^{2} J(q)+m^{2}(q),
$$

where $q^{2} J(q)$ denotes the "kinetic term" of the gluon propagator and $m^{2}(q)$ is the dynamical gluon mass $[7,43,91,93]$.

The ghost-gluon scattering kernel $H_{\nu \mu}^{a b c}(q, p, r)=$ $-g f^{a b c} H_{\nu \mu}(q, p, r)$ is diagrammatically depicted in Fig. 1. The most general tensorial decomposition of $H_{\nu \mu}(q, p, r)$ is given by $[87,94]$

$$
\begin{aligned}
H_{\nu \mu}(q, p, r)= & A_{1} g_{\mu \nu}+A_{2} q_{\mu} q_{\nu}+A_{3} r_{\mu} r_{\nu} \\
& +A_{4} q_{\mu} r_{\nu}+A_{5} r_{\mu} q_{\nu},
\end{aligned}
$$

where the momentum dependence, $A_{i} \equiv A_{i}(q, p, r)$, has been suppressed for compactness. Notice that, at tree level, $H_{\nu \mu}^{(0)}(q, p, r)=g_{\nu \mu}$, so that the form factors assume the values $A_{1}^{(0)}=1$ and $A_{i}^{(0)}=0$, for $i=2, \ldots, 5$.

As mentioned in the Introduction, our interest in the dynamics of $H_{\nu \mu}$ stems mainly from its connection to two of the most fundamental Yang-Mills vertices [86], namely the ghost-gluon vertex, $\Gamma_{\mu}^{a b c}(q, p, r)=-g f^{a b c} \Gamma_{\mu}(q, r, p)$, and the three-gluon vertex, $\boldsymbol{\Gamma}_{\alpha \mu \nu}^{a b c}(q, r, p)=g f^{a b c} \boldsymbol{\Gamma}_{\alpha \mu \nu}(q, r, p)$, where $g$ denotes the gauge coupling, and $q+r+p=0$; both vertices are shown diagrammatically in Fig. 2.

In particular, $H_{\nu \mu}$ and the aforementioned vertices are related by the following STIs:

$$
q^{\nu} H_{\nu \mu}(q, p, r)=\Gamma_{\mu}(q, p, r)
$$

and

$$
\begin{aligned}
r^{\mu} \boldsymbol{\Gamma}_{\alpha \mu \nu}(q, r, p)= & F(r)\left[\Delta^{-1}(q) P_{\alpha}^{\mu}(q) H_{\mu \nu}(q, r, p)\right. \\
& \left.-\Delta^{-1}(p) P_{\nu}^{\mu}(p) H_{\mu \alpha}(p, r, q)\right],
\end{aligned}
$$

where $F(q)$ stands for the ghost dressing function, which is obtained from the ghost propagator, $D^{a b}(q)=\delta^{a b} D(q)$, through

$$
D(q)=\frac{i F(q)}{q^{2}} .
$$

Evidently, the contraction of $\boldsymbol{\Gamma}_{\alpha \mu \nu}(q, r, p)$ with respect to $q^{\alpha}$ or $p^{\nu}$ leads to cyclic permutations of the STI in Eq. (2.5).
Employing the standard tensorial decomposition of $\Gamma_{\mu}(q, p, r)$,

$$
\Gamma_{\mu}(q, p, r)=q_{\mu} B_{1}(q, p, r)+r_{\mu} B_{2}(q, p, r),
$$

where, at tree level, $B_{1}^{(0)}=1$ and $B_{2}^{(0)}=0$, it is straightforward to establish from the STI of Eq. (2.4) that [88]

$$
\begin{aligned}
& B_{1}=A_{1}+q^{2} A_{2}+(q \cdot r) A_{4} ; \\
& B_{2}=(q \cdot r) A_{3}+q^{2} A_{5} .
\end{aligned}
$$

Thus, knowledge of the form factors of $H_{\nu \mu}$ determines fully the corresponding form factors of the ghost-gluon vertex $\Gamma_{\mu}(q, p, r)$.

On the other hand, the extraction of information on the structure of $\boldsymbol{\Gamma}_{\alpha \mu \nu}(q, r, p)$ from Eq. (2.5) (and its permutations) is significantly more involved, both conceptually and operationally. Note in particular that, in the framework composed by the union between the pinch technique and the background field method (PT-BFM) [21], the form of $\boldsymbol{\Gamma}_{\alpha \mu \nu}(q, r, p)$ is intimately connected with the mechanism that is responsible for the infrared finiteness of the gluon propagator, and especially the form employed in Eq. (2.2). Specifically, the full vertex is composed by two characteristic pieces $[90,91]$

$$
\Gamma_{\alpha \mu \nu}(q, r, p)=\Gamma_{\alpha \mu \nu}(q, r, p)+V_{\alpha \mu \nu}(q, r, p),
$$

where the term $V_{\alpha \mu \nu}(q, r, p)$ is very special, in the sense that it contains "longitudinally coupled" massless poles, i.e., has the general form

$$
\begin{aligned}
V_{\alpha \mu \nu}(q, r, p)= & \left(\frac{q_{\alpha}}{q^{2}}\right) A_{\mu \nu}(q, r, p)+\left(\frac{r_{\mu}}{r^{2}}\right) B_{\alpha \nu}(q, r, p) \\
& +\left(\frac{p_{\nu}}{p^{2}}\right) C_{\alpha \mu}(q, r, p)
\end{aligned}
$$

which trigger the Schwinger mechanism and the subsequent emergence of a gluonic mass scale [95]. Note that, by virtue of Eq. (2.10), $V^{\alpha \mu \nu}(q, r, p)$ satisfies the important projection property $P_{\alpha \alpha^{\prime}}(q) P_{\mu \mu^{\prime}}(r) P_{\nu \nu^{\prime}}(p) \times$ $V^{\alpha \mu \nu}(q, r, p)=0$.

As has been explained in detail in the literature mentioned above, the decompositions of $\Delta^{-1}$ and $\boldsymbol{\Gamma}$ put forth in Eqs. (2.2) and (2.9), respectively, prompt a particular realization of Eq. (2.5). Specifically, the initial STI is decomposed into two partial STIs, one for $\Gamma_{\alpha \mu \nu}(q, r, p)$ and one for $V_{\alpha \mu \nu}(q, r, p)$, namely (Minkowski space) $)^{3}$

\footnotetext{
${ }^{3}$ In Minkowski space, $\Delta^{-1}(q)=q^{2} J(q)-m^{2}(q)$; to recover Eq. (2.2), set $q^{2} \rightarrow-q_{\mathrm{E}}^{2}$ and use the transformation conventions of Eq. (5.1); finally, drop the subscript "E."
} 


$$
\begin{aligned}
r^{\mu} \Gamma_{\alpha \mu \nu}(q, r, p)= & F(r)\left[q^{2} J(q) P_{\alpha}^{\mu}(q) H_{\mu \nu}(q, r, p)\right. \\
& \left.-p^{2} J(p) P_{\nu}^{\mu}(p) H_{\mu \alpha}(p, r, q)\right], \\
r^{\mu} V_{\alpha \mu \nu}(q, r, p)= & F(r)\left[m^{2}(p) P_{\nu}^{\mu}(p) H_{\mu \alpha}(p, r, q)\right. \\
& \left.-m^{2}(q) P_{\alpha}^{\mu}(q) H_{\mu \nu}(q, r, p)\right] .
\end{aligned}
$$

The correspondence $\Gamma \leftrightarrow q^{2} J(q)$ and $V \leftrightarrow m^{2}(q)$ leading to Eqs. (2.11) and (2.12) is natural, in the sense that the term $V$ that triggers the generation of the mass saturates, at the same time, the "mass-dependent" part of the STI in Eq. (2.5); however, a comment on its uniqueness is in order (see also footnote 1). In particular, one may envisage the possibility of defining $J^{\prime}(q)=J(q)+f(q)$ and $m^{\prime 2}(q)=m^{2}(q)+h(q)$, such that $\Delta^{-1}(q)=q^{2} J(q)+m^{2}(q)=q^{2} J^{\prime}(q)+m^{\prime 2}(q)$, which forces the constraint $h(q)=-q^{2} f(q)$. The form of $f(q)$, in turn, will be severely constrained by the nonlinear SDEs satisfied by $J(q)$ and $m^{2}(q)$, in conjunction with additional requirements such as the positive-definiteness and monotonicity of the final $\mathrm{m}^{2}(q)$. However, in the absence of a concrete proof stating that $f(q)=0$, the correspondence employed above should be understood as a physically motivated Ansatz.

It turns out that the STI of Eq. (2.12) and its permutations, together with the aforementioned projection property, determine completely the form of $V_{\alpha \mu \nu}(q, r, p)$, which has been worked out in [96].

$\Gamma_{\alpha \mu \nu}(q, r, p)$ contains the bulk of the nonperturbative corrections not related to the poles and is precisely the part that survives when the "transversely projected" vertex $P_{\alpha \alpha^{\prime}}(q) P_{\mu \mu^{\prime}}(r) P_{\nu \nu^{\prime}}(p) \boldsymbol{\Gamma}^{\alpha \mu \nu}(q, r, p)$ is considered. The STI in Eq. (2.11), together with its two cyclic permutations, permits the reconstruction of its longitudinal form factors, through the application of the procedure described in [87]. In practice, the complete construction of the $\mathrm{BC}$ solution depends not only on the infrared behavior of $J$ and $F$, discussed in Sec. IV, but also on the details of $A_{1}, A_{3}$, and $A_{4}$, which are largely unexplored and are the focal point of the present study.

Quite interestingly, the BC construction for the longitudinal part of $\Gamma_{\alpha \mu \nu}$ hinges on the validity of a special relation among $A_{1}, A_{3}$, and $A_{4}$, which in the original work of [87] was shown to hold at the one-loop level (in the Feynman gauge). Subsequently, this relation was derived from the fundamental STI that $H_{\nu \mu}$ satisfies when contracted by the momentum of the incoming gluon [89], and is therefore exact both perturbatively to all orders as well as nonperturbatively. The relation in question may be expressed in terms of the ratio

$$
\mathcal{R}\left(q^{2}, p^{2}, r^{2}\right):=\frac{F(r)\left[A_{1}(q, r, p)+p^{2} A_{3}(q, r, p)+(q \cdot p) A_{4}(q, r, p)\right]}{F(p)\left[A_{1}(q, p, r)+r^{2} A_{3}(q, p, r)+(q \cdot r) A_{4}(q, p, r)\right]},
$$

and states simply that, by virtue of the aforementioned STI, one must have ${ }^{4}$

$$
\mathcal{R}\left(q^{2}, p^{2}, r^{2}\right)=1,
$$

for any value of $q, r$, and $p$.

As we will see in Secs. IV and VI, the constraint of Eq. (2.14) is particularly useful for optimizing the form of the ingredients entering into the computation of the $A_{i}$, and for quantifying the veracity of the truncations and approximations employed.

\section{GHOST-GLUON KERNEL AT THE ONE-LOOP DRESSED LEVEL}

In this section we derive the expressions for the form factors $A_{i}$ within the one-loop dressed approximation. In particular, the four point ghost-gluon scattering amplitude, entering in the diagrammatic definition of $H_{\nu \mu}(q, p, r)$ in Fig. 1, is approximated by its lowest order contributions,

\footnotetext{
${ }^{4}$ An approximate version of this identity was first derived in [97] and further analyzed in [98].
}

including the one-gluon and one-ghost exchange terms, which are subsequently "dressed" as shown in Fig. 3.

Note that the terms kept in the above truncation correspond to the one-particle reducible part of the connected kernel $A^{\mu} A^{\rho} \bar{c} c$ (grey ellipse), while the omitted terms comprise the one-particle irreducible two-ghosttwo-gluon Green's function, whose lowest-order diagrammatic representation may be found in Fig. 6 of [99]. ${ }^{5}$ As was shown in a recent study [100], the inclusion of this subset of corrections into the SDE analysis for $\Gamma_{\mu}$ provides a small contribution of the order of $2 \%$. Therefore, given that $\Gamma_{\mu}$ and $H_{\nu \mu}$ are intimately connected by Eq. (2.4), it is reasonable to expect that the truncation implemented in this work will capture faithfully the main bulk of the result.

Thus, the approximate version of the SDE that we employ reads

$$
H_{\nu \mu}(q, p, r)=g_{\nu \mu}+\left(d_{1}\right)_{\nu \mu}+\left(d_{2}\right)_{\nu \mu}
$$

\footnotetext{
${ }^{5}$ We emphasize that all graphs of that figure are one-loop dressed; therefore, if inserted into the fundamental diagram (first one in Fig. 3), they would give rise to two-loop dressed contributions for $H_{\nu \mu}(q, p, r)$.
} 


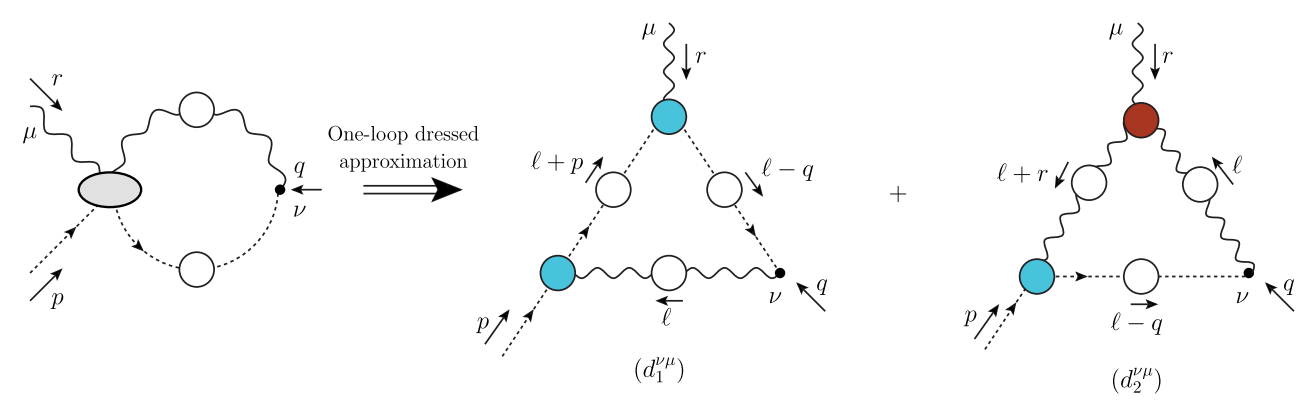

FIG. 3. One-loop dressed approximation of the SDE governing the ghost-gluon scattering kernel.

with

$$
\begin{aligned}
\left(d_{1}\right)_{\nu \mu}= & \frac{1}{2} C_{\mathrm{A}} g^{2} p_{\rho} \int_{\ell} \Delta_{\nu}^{\rho}(\ell) D(\ell+p) D(\ell-q) \\
& \times \Gamma_{\mu}(q-\ell, \ell+p, r) B_{1}(-\ell-p, p, \ell), \\
\left(d_{2}\right)_{\nu \mu}= & \frac{1}{2} C_{\mathrm{A}} g^{2} p_{\rho} \int_{\ell} \Delta_{\nu}^{\beta}(\ell) \Delta^{\alpha \rho}(\ell+r) D(\ell-q) \\
& \times \Gamma_{\mu \alpha \beta}(r,-\ell-r, \ell) B_{1}(q-\ell, p, \ell+r),
\end{aligned}
$$

where $C_{\mathrm{A}}$ is the eigenvalue of the Casimir operator in the adjoint representation, and we have defined the integration measure

$$
\int_{\ell} \equiv \int \frac{\mathrm{d}^{4} \ell}{(2 \pi)^{4}}
$$

Note that in arriving at Eq. (3.2) we have exploited the full transversality of the gluon propagator in the Landau gauge in order to eliminate the $B_{2}$ form factors of two of the ghost-gluon vertices.

It is obvious from Eq. (3.2) that in the soft ghost limit, i.e., $p \rightarrow 0$, the one-loop dressed corrections vanish, i.e., $H_{\nu \mu}(q, p, r)=g_{\nu \mu}$. This result is valid to all orders, independently of the truncation scheme adopted [see, e.g., Eqs. (6.24) and (6.25) of [96] ], being a plain manifestation of Taylor's theorem [101].

The renormalization of Eq. (3.1) proceeds through the replacements [71]

$$
\begin{aligned}
\Delta_{R}\left(q^{2}\right) & =Z_{A}^{-1} \Delta\left(q^{2}\right), \\
F_{R}\left(q^{2}\right) & =Z_{c}^{-1} F\left(q^{2}\right), \\
\Gamma_{R}^{\mu}(q, p, r) & =Z_{1} \Gamma^{\mu}(q, p, r), \\
\Gamma_{R}^{\mu \alpha \beta}(q, r, p) & =Z_{3} \Gamma^{\mu \alpha \beta}(q, r, p), \\
g_{R} & =Z_{g}^{-1} g=Z_{1}^{-1} Z_{A}^{1 / 2} Z_{c} g=Z_{3}^{-1} Z_{A}^{3 / 2} g,
\end{aligned}
$$

where $Z_{A}, Z_{c}, Z_{1}, Z_{3}$, and $Z_{g}$ are the corresponding renormalization constants. Within the momentum subtraction (MOM) scheme that we employ, propagators assume their tree-level values at the subtraction point $\mu$, while an analogous condition is imposed on the vertices, usually implemented at a common value of all their momenta ("symmetric" point).

A well-known consequence of Eq. (2.4) is that $H^{\nu \mu}$ renormalizes as $\Gamma^{\mu}$, namely $H_{R}^{\nu \mu}=Z_{1} H^{\nu \mu}$. The (multiplicative) renormalization of Eq. (3.1) proceeds in the standard way, by replacing the unrenormalized quantities by renormalized ones, using the relations given in Eq. (3.4). Then, it is straightforward to show that the integrands of $\left(d_{1}\right)_{\nu \mu}$ and $\left(d_{2}\right)_{\nu \mu}$ can be written exclusively in terms of the standard renormalization-group invariant quantities formed by $g \Gamma_{\mu} \Delta^{1 / 2} D$ and $g \Gamma^{\nu \sigma \alpha} \Delta^{3 / 2}$; therefore both terms maintain their original form after renormalization. Thus, the renormalized version of Eq. (3.1) reads

$$
H_{R}^{\nu \mu}(q, p, r)=Z_{1}\left[g^{\nu \mu}+\left(d_{1}\right)_{R}^{\nu \mu}+\left(d_{2}\right)_{R}^{\nu \mu}\right],
$$

where the $Z_{1}$ originates from the renormalization of the $H^{\nu \mu}(q, p, r)$ on the left-hand side. The subscript $R$ will subsequently be suppressed to avoid notation clutter.

In what follows we will set $Z_{1}=1$. This particular choice is exact in the case of the soft ghost limit, being strictly enforced by the validity of Taylor's theorem [101]. For any other MOM-related prescription, $Z_{1}$ deviates only slightly (a few percent) from unity, for the subtraction point $\mu=4.3 \mathrm{GeV}$ that we employ. For example, as we have explicitly confirmed from our results, in the case where the MOM prescription is imposed at the symmetric point $\left(p^{2}=r^{2}=q^{2}=\mu^{2}\right)$, instead of the exact $A_{1}(\mu)=1$ we have $A_{1}(\mu)=1.03$.

The relation between $H_{\nu \mu}$ and $\Gamma_{\mu}$, given by Eq. (2.4), prompts a final adjustment, which permits us to preserve the ghost-antighost symmetry at the level of the approximate SDE that we consider. ${ }^{6}$ Specifically, the form factor $B_{1}(q, p, r)$ of the ghost-gluon vertex is symmetric under the exchange of the ghost and antighost momenta, $p$ and $q$, respectively. However, the truncated SDE of Fig. 3 does not respect this special symmetry, because the vertex where the ghost leg is entering is dressed while that of the antighost is

\footnotetext{
${ }^{6}$ This special symmetry of the ghost-gluon vertex is valid only in the Landau gauge [12].
} 
bare. A simple expedient for restoring this property is to "average" the SDEs dressed on either leg [32,33,102], which amounts to substituting into Eq. (3.2)

$$
\begin{aligned}
& B_{1}(-\ell-p, p, \ell) \rightarrow \mathcal{V}_{1}(\ell, q, p, r) \\
& \quad= \frac{1}{2}\left[B_{1}(-\ell-p, p, \ell)+B_{1}(q, \ell-q,-\ell)\right], \\
& B_{1}(q-\ell, p, \ell+r) \rightarrow \mathcal{V}_{2}(\ell, q, p, r) \\
& \quad=\frac{1}{2}\left[B_{1}(q-\ell, p, \ell+r)+B_{1}(q, \ell-q,-\ell)\right] .
\end{aligned}
$$

In general, the individual $A_{i}$ may be projected out from $H_{\nu \mu}(q, p, r)$ by means of a set of suitable projectors, $\mathcal{T}_{i}^{\mu \nu}(q, r)$. In particular,

$$
A_{i}(q, p, r)=\frac{\mathcal{T}_{i}^{\mu \nu}(q, r) H_{\nu \mu}(q, p, r)}{2 h^{2}(q, r)},
$$

where

$$
\begin{aligned}
\mathcal{T}_{1}^{\mu \nu}(q, r)= & h(q, r)\left[h(q, r) g^{\mu \nu}+h^{\mu \nu}(q, r)\right] \\
\mathcal{T}_{2}^{\mu \nu}(q, r)= & -h(q, r) r^{2} g^{\mu \nu}-2 h(q, r) r^{\mu} r^{\nu}-3 r^{2} h^{\mu \nu}(q, r), \\
\mathcal{T}_{3}^{\mu \nu}(q, r)= & \mathcal{T}_{2}^{\mu \nu}(r, q) \\
\mathcal{T}_{4}^{\mu \nu}(q, r)= & h(q, r)(r \cdot q) g^{\mu \nu}+2 h(q, r) q^{\mu} r^{\nu} \\
& +3(r \cdot q) h^{\mu \nu}(q, r) \\
\mathcal{T}_{5}^{\mu \nu}(q, r)= & \mathcal{T}_{4}^{\mu \nu}(r, q),
\end{aligned}
$$

and

$$
\begin{aligned}
h(q, r) & =q^{2} r^{2}-(q \cdot r)^{2}, \\
h^{\mu \nu}(q, r) & =(q \cdot r)\left[q^{\mu} r^{\nu}+q^{\nu} r^{\mu}\right]-r^{2} q^{\mu} q^{\nu}-q^{2} r^{\mu} r^{\nu} .
\end{aligned}
$$

Clearly, since in the present work $H_{\nu \mu}(q, p, r)$ is approximated by Eq. (3.1), the corresponding form factors will be obtained through

$$
A_{i}(q, p, r)=\frac{\mathcal{T}_{i}^{\mu \nu}(q, r)\left[g_{\nu \mu}+\left(d_{1}\right)_{\nu \mu}+\left(d_{2}\right)_{\nu \mu}\right]}{2 h^{2}(q, r)} .
$$

The implementation of the above projections may be carried out using an algebraic manipulation program, such as the Mathematica PACKAGE-X [103,104]; the rather lengthy expressions produced from these projections are presented in Appendix B.

\section{INPUTS AND APPROXIMATIONS}

For the evaluation of Eq. (3.2) we need the following ingredients: (i) the gluon propagator $\Delta(q)$ and its "kinetic" term $J(q)$; (ii) the ghost dressing function $F(q)$; (iii) the three-gluon vertex, entering in $\left(d_{2}\right)_{\nu \mu}$; (iv) the ghost-gluon vertex, entering in both $\left(d_{1}\right)_{\nu \mu}$ and $\left(d_{2}\right)_{\nu \mu}$; and (v) the value of the strong coupling $\alpha_{s} \equiv g^{2} / 4 \pi$ at the renormalization scale $\mu$. The corresponding input quantities will be denoted by $\Delta_{\text {in }}(q), J_{\text {in }}(q), F_{\text {in }}(q), \Gamma_{\mu \alpha \beta}^{\text {in }}$, and $B_{1}^{\text {in }}(Q)$, respectively. It is important to comment already at this point on a characteristic feature shared by inputs (i)-(iv), which is implemented in order for the resulting $A_{i}$ to satisfy Eq. (2.14) as accurately as possible. In particular, in the deep ultraviolet all aforementioned quantities will be forced to tend to their tree-level values; i.e., their one-loop perturbative corrections (logarithms and/or constants) will be suppressed. This, in turn, will guarantee that, for large values of the momenta, the emerging $A_{i}$ will correctly capture their one-loop perturbative behavior (see also discussion in Sec. VI). In what follows we briefly review how the above input quantities are obtained.

(i) and (ii): As was done in a series of previous works $[25,41,90]$, for $\Delta_{\text {in }}(q)$ and $F_{\text {in }}(q)$ we employ fits to the numerical solutions of the corresponding SDEs, which are in excellent agreement with the quenched $\mathrm{SU}(3)$ lattice data of [54], subject to the particular ultraviolet adjustments mentioned above. Below we consider the individual cases (i) and (ii) separately.

(i): The fit for $\Delta_{\text {in }}(q)$ (in Euclidean space) is given by [95]

$$
\Delta_{\text {in }}^{-1}(q)=q^{2} J_{\text {in }}(q)+m^{2}(q),
$$

where the kinetic term has the form

$$
\begin{aligned}
J_{\text {in }}(q)= & 1+\frac{C_{\mathrm{A}} \alpha_{s}}{4 \pi}\left(\frac{\tau_{1}}{q^{2}+\tau_{2}}\right) \\
& \times\left[2 \ln \left(\frac{q^{2}+\rho_{l} m^{2}(q)}{\mu^{2}}\right)+\frac{1}{6} \ln \left(\frac{q^{2}}{\mu^{2}}\right)\right],
\end{aligned}
$$

while the effective gluon mass $m^{2}(q)$ obeys a power-law running $^{7}$ [93],

$$
m^{2}(q)=\frac{m_{0}^{2}}{1+q^{2} / \rho_{m}^{2}},
$$

with the adjustable parameters given by $\tau_{1}=12.68$, $\tau_{2}=1.05 \mathrm{GeV}^{2}, m_{0}^{2}=0.15 \mathrm{GeV}^{2}, \rho_{m}^{2}=1.18 \mathrm{GeV}^{2}$, and $\rho_{l}=102.3$. In the left panel of Fig. 4 we show the lattice data for $\Delta(q)$ (circles) [54], together with the corresponding fit (blue continuous curve) given by the combination of Eqs. (4.1)-(4.3).

\footnotetext{
${ }^{7}$ The solutions for $m^{2}(q)$ found in [93] deviate slightly from the exact power law running, in compliance with the operator product expansion (see also [105]). In particular, $m^{2}(q)=m_{0}^{2} /$ $\left[1+\left(q^{2} / \rho_{m}^{2}\right)^{1+\gamma}\right]$, with $\gamma$ ranging between 0.1 and 0.3 , depending on a number of subtle assumptions and approximations. Here we use for simplicity the case $\gamma=0$; the dependence of our results on variations of $\gamma$ (within the aforementioned range of values) is negligible.
} 

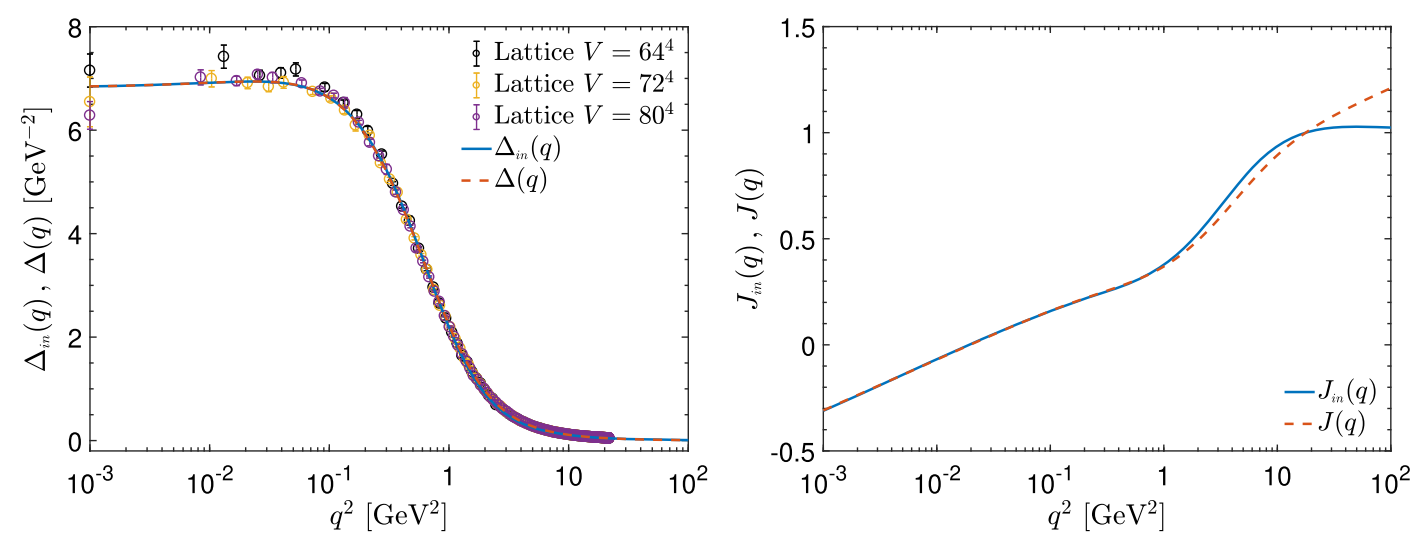

FIG. 4. The fits for $\Delta_{\text {in }}(q)$ (left panel) and $J_{\text {in }}(q)$ (right panel) given by Eqs. (4.1) and (4.2), respectively (blue continuous curves). The fits for $\Delta(q)$ and $J(q)$ follow the same functional dependence but with $\tau_{1} /\left(q^{2}+\tau_{2}\right) \rightarrow 1$. The lattice data are from Ref. [54].

On the right panel of Fig. 4 we present the $J_{\text {in }}(q)$ of Eq. (4.2); the reason for displaying it in isolation is that it constitutes the main ingredient in the approximation implemented for the three-gluon vertex in item (iii) [see Eqs. (4.5) and (4.6)]. Notice that the $J_{\text {in }}(q)$ contains both massive and massless logarithms, which are crucial for triggering three characteristic features, namely its suppression with respect to its tree-level value $\left[J^{(0)}(q)=1\right]$ for a wide range of physically relevant momenta, the reversal of its sign (zero crossing), and its logarithmic divergence at the origin $[34,68]$. These features, in turn, will be inherited by the components of the three-gluon vertex constructed in (iii). Even though $J_{\text {in }}(q)$ contains these logarithms, for large $q^{2}$ it tends to 1 , in compliance with the requirement discussed above, due to the inclusion of the function $\tau_{1} /\left(q^{2}+\tau_{2}\right)$; note that this function becomes 1 in the "bona-fide" fit for $J(q)$, which is also displayed in Fig. 4, for direct comparison.

(ii): The fit for $F_{\text {in }}(q)$ is shown in the left panel of Fig. 5 (blue continuous line), together with the corresponding lattice data; its functional form is given by

$$
F_{\text {in }}(q)=1+\frac{\sigma_{1}}{q^{2}+\sigma_{2}},
$$

with $\sigma_{1}=0.70 \mathrm{GeV}^{2}$ and $\sigma_{2}=0.39 \mathrm{GeV}^{2}$. Again, in the limit of large $q^{2}$, the above expression recovers the treelevel result, i.e., $F_{\text {in }}(q)=1$. In that same plot, the red dashed line corresponds to the fit of $F(q)$ introduced in Eq. (6.1), which corresponds to the typical solution of the SDE for $F(q)$ [71], and, as such, contains the appropriate perturbative logarithms. Evidently, the difference between the two fits becomes relevant in the deep ultraviolet, where the $F(q)$ of Eq. (6.1) deviates gradually from unity, eventually approaching zero at a logarithmic rate.

(iii) and (iv): The fully dressed vertex $\Gamma_{\mu \alpha \beta}$ and $\Gamma_{\mu}$ enter in Eq. (3.2) that controls $H_{\nu \mu}$, but, at the same time, the determination of their longitudinal parts from Eqs. (2.11) and (2.8) requires the knowledge of $H_{\nu \mu}$, converting the problem into an extended system of coupled equations. ${ }^{8}$ However, given the complexity of such an endeavor, we will employ instead a set of approximations for these two vertices. We next analyze (iii) and (iv) separately.

(iii): Let us first consider the three-gluon vertex, entering in $\left(d_{2}\right)_{\nu \mu}$, and set $t=-(\ell+r)$. Our way of approximating $\Gamma_{\mu \alpha \beta}(r, t, \ell)$ is the following. First, we consider the STIs exemplified by Eq. (2.11), and "abelianize" them by turning off the ghost sector, i.e., setting $F(r)=1$ and $H_{\nu \mu}=g_{\nu \mu}$. The resulting identities may then be "solved" following the $\mathrm{BC}$ procedure [87], thus furnishing the abelianized longitudinal form factors, $X_{i}(i=1 \ldots 10$, which contain combinations of the function $J(q)$ only. Then, the "input" $\Gamma_{\mu \alpha \beta}(r, t, \ell)$, to be denoted by $\Gamma_{\mu \alpha \beta}^{\text {in }}(r, t, \ell)$, is chosen to contain only the three tensorial structures that comprise the tree-level vertex, multiplied by the corresponding form factors, which are related to each other by the Bose symmetry. In particular,

$$
\begin{aligned}
\Gamma_{\mu \alpha \beta}^{\mathrm{in}}(r, t, \ell)= & (r-t)_{\beta} g_{\mu \alpha} X_{1}^{\mathrm{in}}(r, t, \ell) \\
& +(t-\ell)_{\mu} g_{\alpha \beta} X_{1}^{\mathrm{in}}(t, \ell, r) \\
& +(\ell-r)_{\alpha} g_{\beta \mu} X_{1}^{\mathrm{in}}(\ell, r, t),
\end{aligned}
$$

with

$$
X_{1}^{\text {in }}(r, t, \ell)=\frac{1}{2}\left[J_{\text {in }}(r)+J_{\text {in }}(t)\right] .
$$

Notice that at tree level $X_{1}^{\text {in }}=1$, and Eq. (4.5) reduces indeed to

$$
\Gamma_{\mu \alpha \beta}^{(0)}(r, t, \ell)=(r-t)_{\beta} g_{\mu \alpha}+(t-\ell)_{\mu} g_{\alpha \beta}+(\ell-r)_{\alpha} g_{\beta \mu} .
$$

\footnotetext{
${ }^{8}$ It should be clear that a fully self-consistent treatment would also require information on the transverse parts of the vertices, which are a priori inaccessible to a gauge-technique based approach.
} 

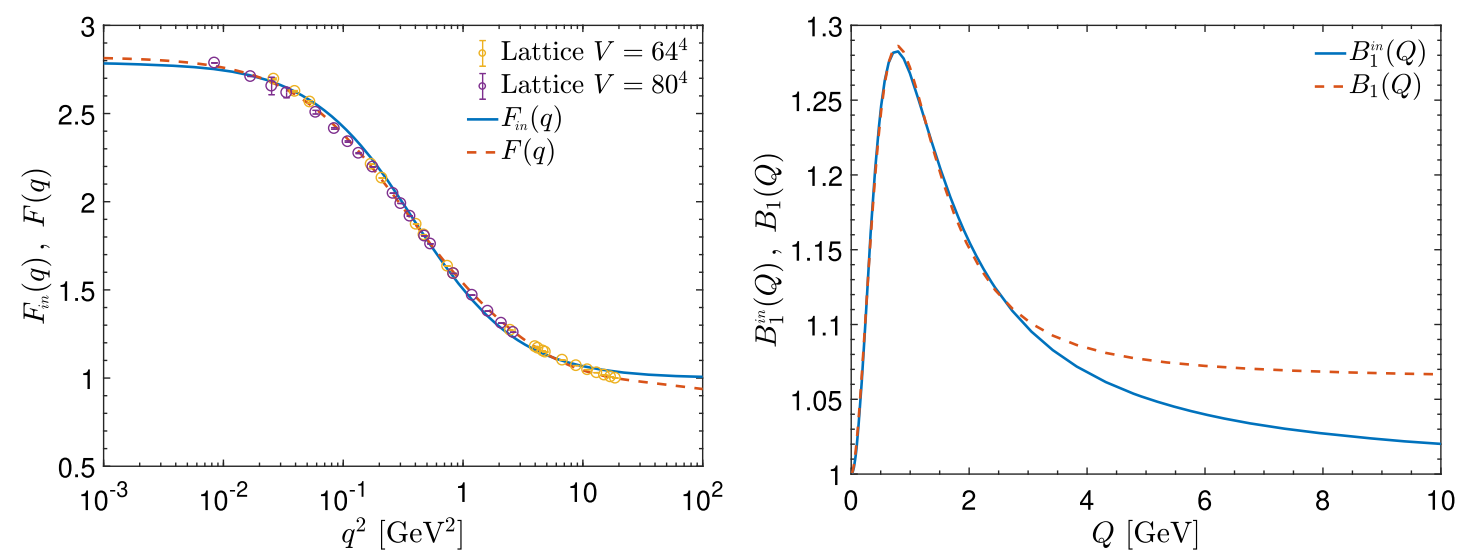

FIG. 5. Left panel: Fits for $F(q)$ without ultraviolet logarithms (blue continuous line), corresponding to Eq. (4.4), and with ultraviolet logarithms (red dashed line), given by Eq. (6.1), compared to the lattice data from [54]. Right panel: The form factor $B_{1}^{\text {in }}(Q)$ given by Eq. (4.9) (blue continuous line), and its counterpart $B_{1}(Q)$ with the one-loop correction (red dashed line).

Thus, the $\Gamma_{\mu \alpha \beta}^{\mathrm{in}}$ of Eq. (4.5) will be used as a "seed" for obtaining the one-loop dressed approximation for $H_{\nu \mu}$. Note that, in addition to the remaining seven longitudinal form factors that have not been included in $\Gamma_{\mu \alpha \beta}^{\text {in }}$ for simplicity, the uncertainties associated with the omission of all transverse structures must also be kept in mind.

(iv): Turning to the ghost-gluon vertex, as mentioned right after Eq. (3.3), two out of the three vertices have been naturally replaced by their $B_{1}$ components, and only the $\Gamma_{\mu}(q-\ell, \ell+p, r)$ in $\left(d_{1}\right)_{\nu \mu}$ contains both $B_{1}$ and $B_{2}$. In what follows we will set (by hand) $B_{2}=0$ for this vertex and retain only $B_{1}$; thus, at this point, all ghost-gluon vertices appearing in the problem have been replaced by their $B_{1}$ form factor.

The approximation used for $B_{1}(q, p, r)$ is obtained as follows. We start by carrying out the first iteration of Eq. (3.2), using for $B_{1}$ its tree-level value. This furnishes the first approximation for the $A_{i}(q, p, r)$, which, by means of the first relation in Eq. (2.8), yields the next approximation for $B_{1}(q, p, r)$. At this point we isolate from $B_{1}(q, p, r)$ the "slice" that corresponds to the "totally symmetric" configuration

$q^{2}=p^{2}=r^{2}=Q^{2}, \quad q \cdot p=q \cdot r=p \cdot r=-\frac{1}{2} Q^{2}$,

shown in the right panel of Fig. 5 (red dashed line). Then, to get $B_{1}^{\text {in }}(Q)$ we adjust the "tail" of the curve, such that it reaches the tree-level value 1 for large $Q$; the resulting functional form may be fitted by

$$
B_{1}^{\text {in }}(Q)=1+\frac{\tau_{1} Q^{2}}{\left(1+\tau_{2} Q^{2}\right)^{\lambda}},
$$

where the parameters $\tau_{1}=2.21 \mathrm{GeV}^{-2}, \tau_{2}=2.50 \mathrm{GeV}^{-2}$, and $\lambda=1.68$. Past this point, the iterative procedure described above is discontinued, and the $B_{1}^{\text {in }}(Q)$ of Eq. (4.9) is fixed as the final input in Eq. (3.2).

After the above simplification, Eq. (3.6) becomes

$$
\begin{aligned}
& \mathcal{V}_{1}(\ell, q, p, r)=B_{1}^{\text {in }}(\ell), \\
& \mathcal{V}_{2}(\ell, q, p, r)=\frac{1}{2}\left[B_{1}^{\text {in }}(\ell+r)+B_{1}^{\text {in }}(\ell)\right] .
\end{aligned}
$$

(v): Finally, for most of the analysis, the strong charge will assume the value $\alpha_{s}=0.22$ at the subtraction point $\mu=4.3 \mathrm{GeV}$, where all Green's functions are renormalized. The determination of this particular value is rather convoluted, involving the combination of four-loop perturbative results, nonperturbative information included in the vacuum condensate of dimension two, and the extraction of $\Lambda_{\mathrm{QCD}}$ from lattice results of the ghost-gluon vertex in the Taylor kinematics [106]. Given the theoretical uncertainties associated with some of the aforementioned ingredients, we consider the value $\alpha_{s}=0.22$ rather approximate; in fact, as we will see in Sec. VII, the final analysis seems to favor slightly higher values of the charge, of the order of $\alpha_{s}=0.25$. Note that the difference between using $\alpha_{s}=0.22$ or $\alpha_{s}=0.25$ is practically imperceptible at the level of the 3D plots presented in the next section; however, it becomes visible when particular "slices" are isolated (see left panel of Fig. 16).

\section{RESULTS FOR THE FORM FACTORS OF THE GHOST-GLUON KERNEL}

In this section we present the results for the five form factors $A_{i}$. We will first present 3D plots in general Euclidean kinematics, and then we take a closer look at three special kinematic limits.

\section{A. 3D plots}

First, we use the standard conversion rules to pass Eq. (3.2) and its ingredients from Minkowski to 
Euclidean space [41]. In particular, $\left(q^{2}, p^{2}, q \cdot p\right) \rightarrow$ $-\left(q_{\mathrm{E}}^{2}, p_{\mathrm{E}}^{2}, q_{\mathrm{E}} \cdot p_{\mathrm{E}}\right)$, and

$$
\begin{aligned}
& \Delta\left(q^{2}\right) \stackrel{q^{2} \rightarrow-q_{\mathrm{E}}^{2}}{\longrightarrow}-\Delta_{\mathrm{E}}\left(q_{\mathrm{E}}^{2}\right) ; \quad D\left(q^{2}\right) \stackrel{q^{2} \rightarrow-q_{\mathrm{E}}^{2}}{\longrightarrow}-D_{\mathrm{E}}\left(q_{\mathrm{E}}^{2}\right) ; \\
& B_{i}(q, p, r) \rightarrow B_{i}\left(q_{\mathrm{E}}, p_{\mathrm{E}}, r_{\mathrm{E}}\right) ; \\
& \quad A_{j}(q, p, r) \rightarrow A_{j}\left(q_{\mathrm{E}}, p_{\mathrm{E}}, r_{\mathrm{E}}\right),
\end{aligned}
$$

for $i=1,2, j=1, \ldots, 5$.

In addition, the measure defined in Eq. (3.3) becomes

$$
\int_{\ell}=i \int_{\ell_{\mathrm{E}}}
$$

which in spherical coordinates is given by

$$
\begin{aligned}
\int_{\ell_{\mathrm{E}}}= & \frac{1}{32 \pi^{4}} \int_{\Lambda_{\mathrm{IR}}^{2}}^{\Lambda_{\mathrm{UV}}^{2}} \mathrm{~d} \ell_{\mathrm{E}}^{2} \ell_{\mathrm{E}}^{2} \int_{0}^{\pi} \mathrm{d} \phi_{1} \sin ^{2} \phi_{1} \\
& \times \int_{0}^{\pi} \mathrm{d} \phi_{2} \sin \phi_{2} \int_{0}^{2 \pi} \mathrm{d} \phi_{3} .
\end{aligned}
$$

Note that, for numerical purposes, we have introduced in the radial integration an infrared and ultraviolet cutoffs $\Lambda_{\mathrm{IR}}^{2}$ and $\Lambda_{\mathrm{UV}}^{2}$, respectively; their numerical values will fix the overall size of our numerical grid, namely $\left[5 \times 10^{-5} \mathrm{GeV}^{2}\right.$, $\left.5 \times 10^{3} \mathrm{GeV}^{2}\right]$.

A standard choice for the orientation of the Euclidean four-momenta $q$ and $p$ and the integration momentum $\ell$ is (from now on we suppress the subscript "E")

$$
\begin{aligned}
q= & |q|(1,0,0,0) ; \\
p= & |p|(\cos \theta, \sin \theta, 0,0) ; \\
\ell= & |\ell|\left(\cos \phi_{1}, \sin \phi_{1} \cos \phi_{2}, \sin \phi_{1} \sin \phi_{2} \cos \phi_{3},\right. \\
& \left.\sin \phi_{1} \sin \phi_{2} \sin \phi_{3}\right) .
\end{aligned}
$$

Evidently, $q^{2}=|q|^{2}, p^{2}=|p|^{2}$, and $q \cdot p=|q||p| \cos \theta$.

In what follows we will express all relevant form factors as functions of $q^{2}, p^{2}$, and the angle $\theta$, namely $A_{i}(q, p, r) \rightarrow A_{i}\left(q^{2}, p^{2}, \theta\right)$. Note also that since the quantities entering in the integrals do not depend on the angle $\phi_{3}$, the last integral in (5.3) furnishes simply a factor of $2 \pi$.

The evaluation of the ghost-gluon scattering kernel given by Eqs. (3.1) and (3.2) amounts to a three-dimensional integration for each combination of external momenta and angles, namely $\left(q^{2}, p^{2}, \theta\right)$, and for each of the five $A_{i}$. These integrations were performed numerically with the adaptative algorithm of Ref. [107], employing an 11th degree polynomial rule. The results were computed with the external squared momenta distributed logarithmically on a grid with 80 points, in the range $\left[5 \times 10^{-5} \mathrm{GeV}^{2}\right.$, $5 \times 10^{3} \mathrm{GeV}^{2}$ ], whereas for the angle $\theta$ the grid was composed of 19 uniformly distributed points within $[0, \pi]$.
In Figs. 6 and 7, we present a typical set of results for the form factors $A_{i}$, for $\theta=0$ and $\theta=\pi$.

It is important to notice that all form factors exhibit the following common features: (i) in the infrared, they display considerable departures from their tree-level values; (ii) in the ultraviolet they approach the corresponding one-loop answers, given in Appendix $A^{9}$; (iii) in general, they display a mild dependence on the angle $\theta$.

Moreover, we find that $A_{1}$ is finite in the infrared, whereas $A_{2}, A_{3}, A_{4}$, and $A_{5}$ diverge logarithmically. The origin of these divergences may be traced back to two different sources: (i) the massless of the ghost propagators appearing as $\left(d_{1}\right)_{\nu \mu}$ of Fig. 3, or (ii) the "unprotected" logarithms contained in the $J_{\text {in }}(q)$ that enter in the Ansatz of $\Gamma_{\mu \alpha \beta}^{\text {in }}$ given in Eq. (4.5), thus altering the behavior of the graph $\left(d_{2}\right)_{\nu \mu}$.

In the next subsection, we will carefully scrutinize the circumstances leading to the aforementioned infrared logarithmic divergences, for each one of the four form factors.

\section{B. Special kinematics limits}

In this subsection we first extract from the general 3D solutions for the $A_{i}$ reported above three special kinematic configurations, corresponding to particular 2D slices. Then, we compare them with (i) the corresponding perturbative expressions computed at one loop; (ii) the oneloop massive results, obtained by using "naive" massive gluon propagators inside the one-loop diagrams (see Appendix A); and (iii) the results found when the threegluon vertex appearing in $\left(d_{2}\right)_{\mu \nu}$ is kept at its tree-level value, i.e., setting $X_{1}^{\text {in }}(r, t, \ell)=1$. As we will see, the comparisons (ii) and (iii) are fundamental for identifying the origin of the infrared logarithmic divergences displayed by the four $A_{i}$. Specifically, by means of the one-loop massive calculation one can establish analytically whether $\left(d_{1}\right)_{\mu \nu}$ and $\left(d_{2}\right)_{\mu \nu}$ are individually convergent or divergent, depending on the nature of the propagators comprising them. As for (iii), the use of $\Gamma^{(0)}$ instead of $\Gamma^{\text {in }}$ helps us identify the dressing of the latter as the only reason for the infrared divergences encountered in $\left(d_{2}\right)_{\mu \nu}$.

Thus, through this entire subsection, we display four curves in all panels. The curves correspond to the full case (2D slices) [using $\Gamma^{\mathrm{in}}$ ] (blue continuous line), the one-loop result (purple dotted line), the one-loop massive with $m^{2}=$ $0.15 \mathrm{GeV}^{2}$ (green dash-dotted line), and the case where the

\footnotetext{
${ }^{9}$ This particular property is expected, given that the input functions have been adjusted precisely to that purpose, as discussed in the previous section. Note, however, that possible deviations from this prescribed behavior may be produced, due to artifacts of the numerical treatment (see discussion in the third paragraph of the soft gluon limit in Sec. V B).
} 

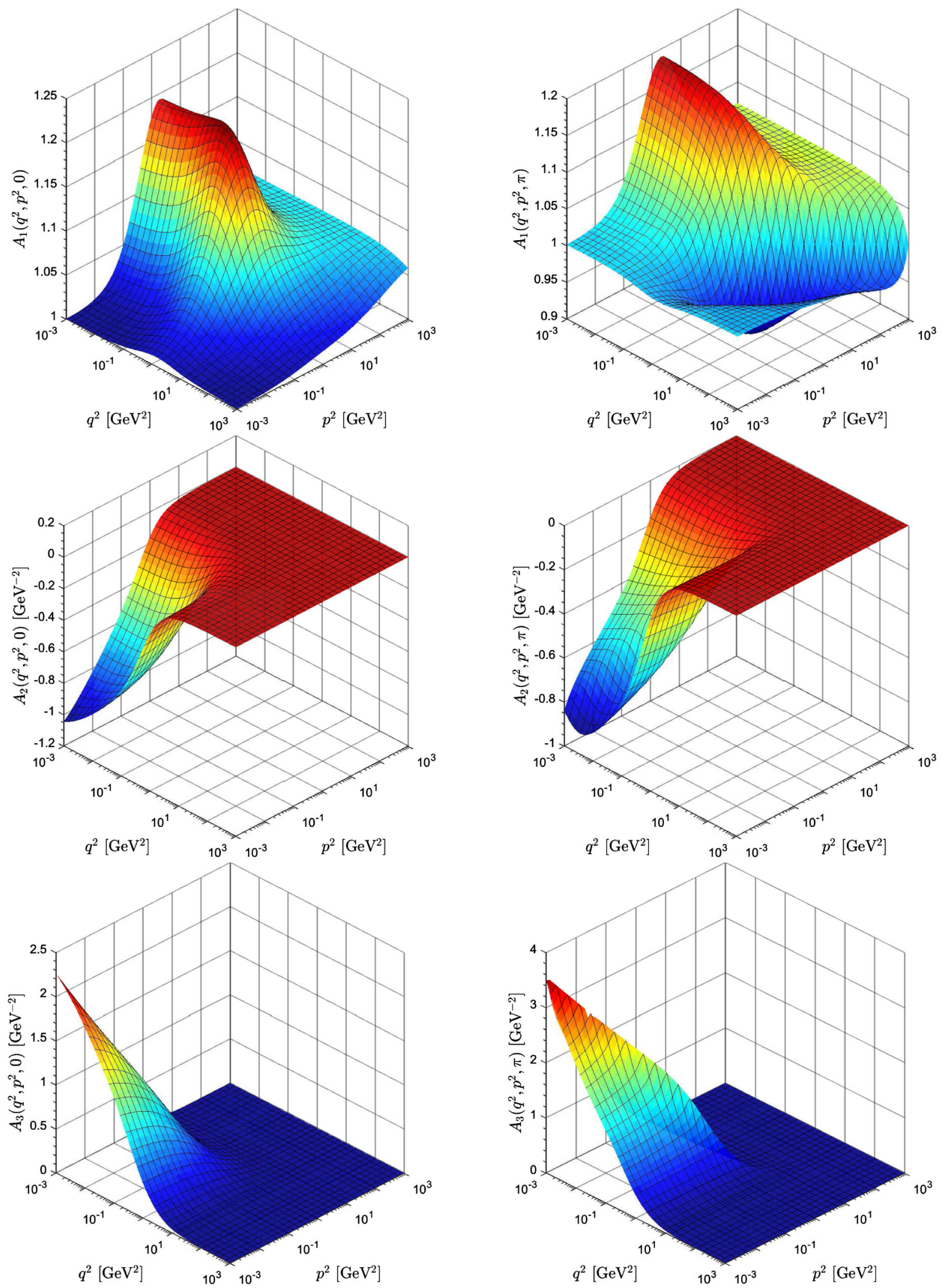

FIG. 6. The form factors of the ghost-gluon scattering kernel $A_{1}\left(q^{2}, p^{2}, \theta\right)$ (first row), $A_{2}\left(q^{2}, p^{2}, \theta\right)$ (second row), and $A_{3}\left(q^{2}, p^{2}, \theta\right)$ (third row) for $\theta=0, \theta=\pi$, and $\alpha_{s}=0.22$. 

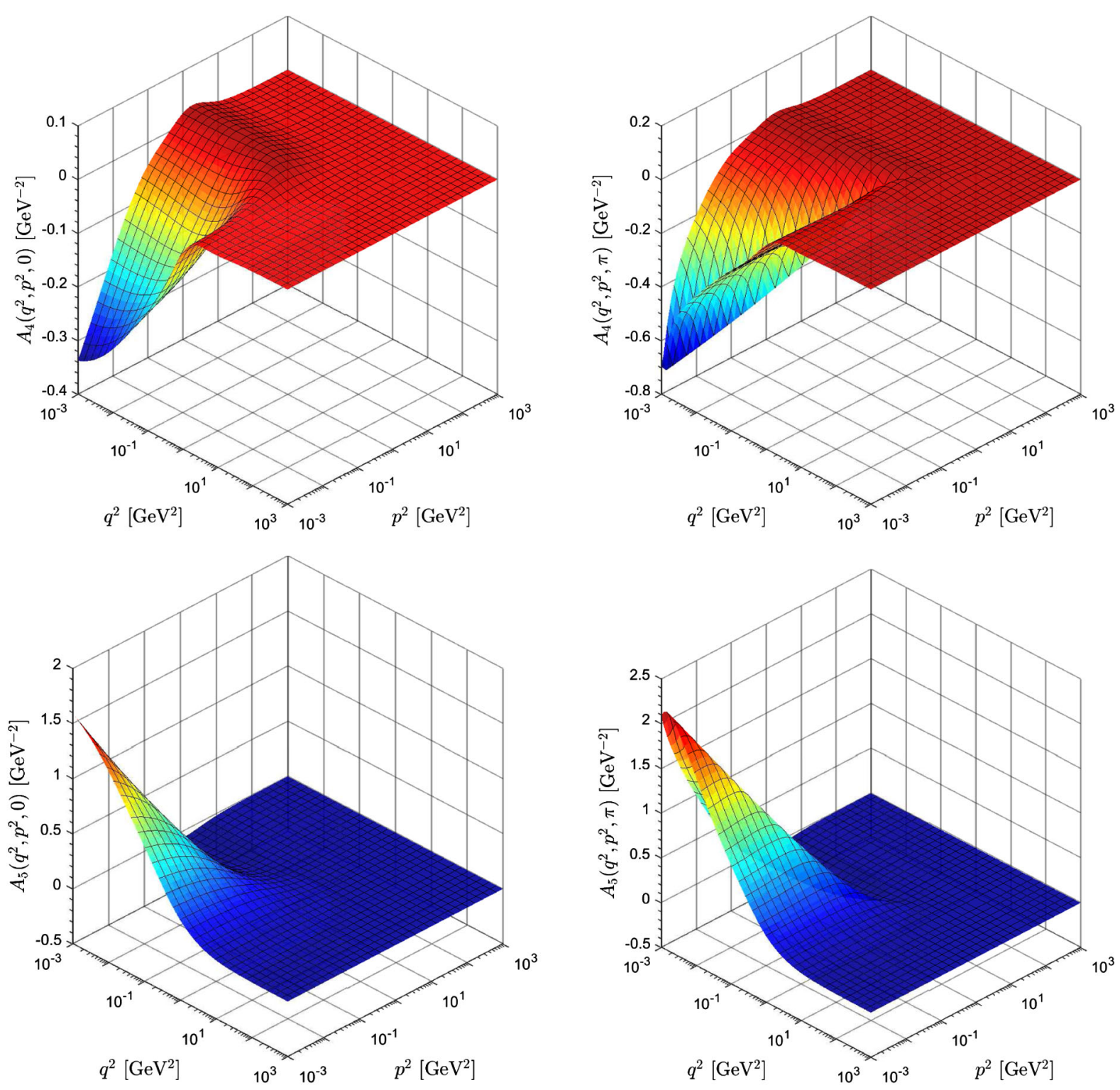

FIG. 7. The form factors of the ghost-gluon scattering kernel $A_{4}\left(q^{2}, p^{2}, \theta\right)$ (top row) and $A_{5}\left(q^{2}, p^{2}, \theta\right)$ (bottom row) for $\theta=0, \theta=\pi$, and $\alpha_{s}=0.22$.

$\Gamma_{\mu \alpha \beta}^{(0)}$ of Eq. (4.7) is used as the input in $\left(d_{2}\right)_{\mu \nu}$ (red dashed line). We adopt the same color convention in all panels.

Before proceeding, let us emphasize that, in order to expedite the one-loop calculations, we have implemented the corresponding kinematic limits directly at the level of $H_{\nu \mu}$, i.e., before projecting out the corresponding form factors. As a result, and depending on the details of the limit considered, certain tensorial structures, together with the accompanying form factors, are completely eliminated from the decomposition of $H_{\nu \mu}$ given in Eq. (2.3). Of course, the form factors that are eliminated are nonvanishing, as may easily be verified from the appropriate slices of the corresponding 3D plots. (i) The soft gluon limit, which means that $r=0$; then, the momenta $q$ and $p$ have the same magnitude, $|p|=|q|$, and are antiparallel, i.e., $\theta=\pi$. Our results are expressed in terms of the momentum $q$.

When this kinematic limit is implemented as described above, the only tensorial structures that survive are those associated with $A_{1}$ and $A_{2}$ [see Eq. (2.3)]. These two form factors are shown in Fig. 8. $A_{1}(q,-q, 0)$ (left panel) displays only a mild deviation from its tree-level value in the entire range of momenta. The maximum deviation is of the order of $5 \%$, and is located around $q \approx 1 \mathrm{GeV}$. It is interesting to observe that the one-loop massive and the nonperturbative calculation with $\Gamma_{\mu \alpha \beta}^{(0)}$ also display the peak around the same region of momenta, although there is a 

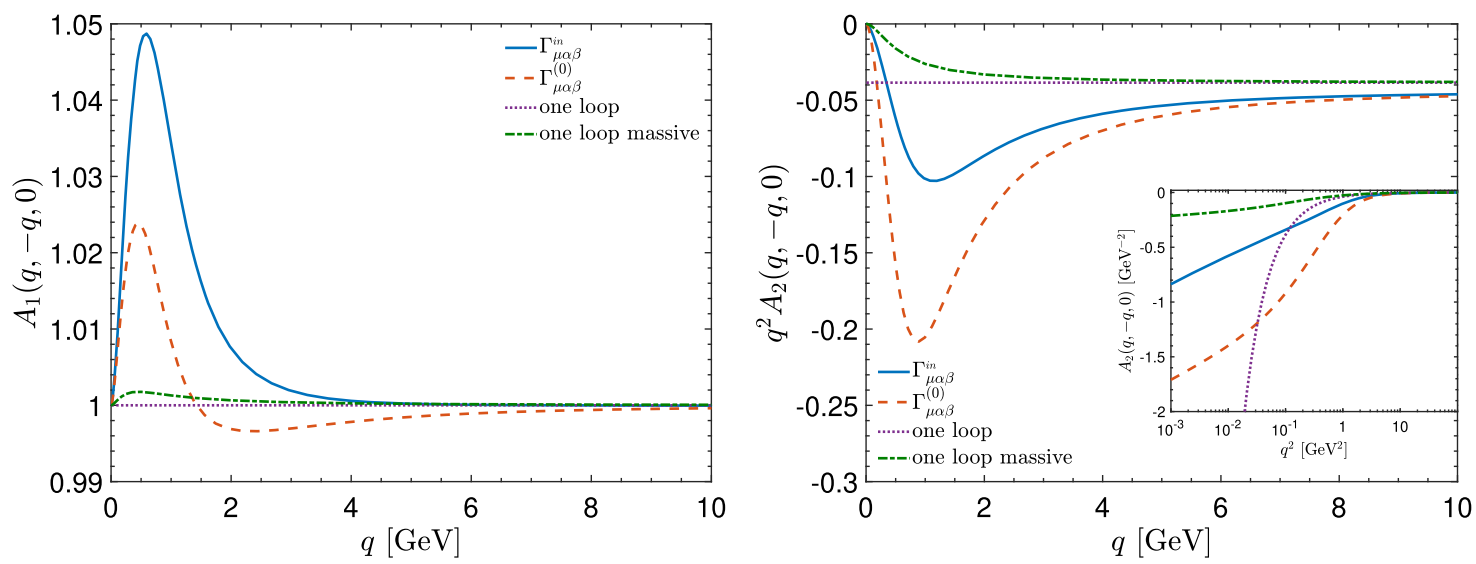

FIG. 8. (Soft gluon kinematics) Left: Comparison between the $A_{1}(q,-q, 0)$ computed using $\Gamma_{\mu \alpha \beta}^{\text {in }}$ (blue continuous line) and the one obtained when $\Gamma_{\mu \alpha \beta}^{(0)}$ is used instead (red dashed line). The massless (purple dotted line) and the massive (green dash-dotted line) oneloop perturbative results are given by Eqs. (A5) and (A6), respectively. Right: Same comparison for the dimensionless combination $q^{2} A_{2}(q,-q, 0)$. In the inset we show the corresponding logarithmic infrared divergence of $A_{2}(q,-q, 0)$, using a logarithmic scale for $q^{2}$. Note that the purple dotted curve shows a much steeper (linear) divergence.

clear quantitative difference in their heights. Notice that $A_{1}$ is infrared finite, and for all curves we have $A_{1}(0,0,0)=1$. This particular value is recovered again for high values of $q$, as expected from the one-loop calculation of Eq. (A5); one may clearly observe how all curves approach each other and practically coincide around $q \approx 10 \mathrm{GeV}$.

It is important to mention that, in the above analysis, the limit $\theta=\pi$ is rather subtle. This happens because the projectors of the $A_{i}$ introduce a $\sin ^{4} \theta$ in their denominator [see Eq. (3.10)], whose cancellation requires the proper Taylor expansion of the numerator around $\sin \theta \approx 0$. If instead of expanding one were to use a configuration whose angle was slightly different from $\pi$, the resulting curve would fail to approach the one-loop result, running instead "parallel" to it.

On the right panel of Fig. 8 we show the dimensionless combination $q^{2} A_{2}(q,-q, 0)$, which in the ultraviolet tends toward the constant value predicted by the one-loop result given by Eq. (A5). Once again, the maximum deviation from its tree-level value is located around $q \approx 1 \mathrm{GeV}$, and the nonperturbative calculation with $\Gamma_{\mu \alpha \beta}^{(0)}$ captures rather well the position of this minimum, although its depth is bigger. In order to make apparent the infrared logarithmic divergence, in the inset we show the dimensionful $A_{2}(q,-q, 0)$ alone, using a logarithmic scale. Notice that the one-loop massive analytical result [see Eq. (A7)] and the nonperturbative calculation with $\Gamma_{\mu \alpha \beta}^{(0)}$ also display the same type of divergence in the infrared. In addition, observe that the use of $\Gamma_{\mu \alpha \beta}^{\text {in }}$ slows down the rate of the negative infrared divergence of $A_{2}$. It is interesting to mention that the infrared divergence of $A_{2}$ is due to the presence of the two massless ghost propagators in the diagrams $\left(d_{1}\right)$ of Fig. 3. In Table I we summarize how each diagram behaves in the infrared separately for the cases presented in the plot, except for the pure perturbative oneloop calculation.

(ii) The soft antighost limit, in which $q=0$ and the momenta $|p|=|r|$; evidently, $|q||p| \cos \theta=0$, and any dependence on the angle $\theta$ is washed out.

In this limit, we may recover information only about $A_{1}$ and $A_{3}$, which depend on a unique momentum, namely $r$. In Fig. 9, we can see that both form factors, $A_{1}(0,-r, r)$ and $A_{3}(0,-r, r)$, display a sizable deviation from their treelevel expressions around the region $r \approx 1.0-1.5 \mathrm{GeV}$. Moreover, in the ultraviolet they are approaching the one-loop results of Eq. (A9). $A_{1}$ is again infrared finite, while $A_{3}$ is logarithmically divergent, as shown in the inset. Note that the one-loop massive result [see Eq. (A11)] and

TABLE I. The summary of the infrared limits of the individual contributions of the diagrams $\left(d_{1}\right)$ and $\left(d_{2}\right)$ appearing in Fig. 3. The limits are for (i) the one-loop massive results [see Eqs. (A7), (A11), and (A16)]; (ii) the nonperturbative result obtained when $\Gamma_{\mu \alpha \beta}^{(0)}$ is used as input in the diagram $\left(d_{2}\right)$; and (iii) the nonperturbative result obtained with $\Gamma_{\mu \alpha \beta}^{\text {in }}$. The letter " $F$ " stands for "finite" and the acronym "LD" for "logarithmically divergent."

\begin{tabular}{|c|c|c|c|c|c|c|c|}
\hline \multirow[b]{2}{*}{ Form factors } & \multicolumn{2}{|c|}{ One-loop massive } & \multicolumn{2}{|c|}{$\Gamma_{\mu \alpha \beta}^{(0)}$} & \multicolumn{3}{|c|}{$\Gamma_{\mu \alpha \beta}^{\text {in }}$} \\
\hline & $\left(d_{1}\right)$ & $\left(d_{2}\right)$ & $\left(d_{1}\right)$ & $\left(d_{2}\right)$ & $\left(d_{1}\right)$ & $\left(d_{2}\right)$ & Total \\
\hline$A_{1}$ & $\mathrm{~F}$ & $\mathrm{~F}$ & $\mathrm{~F}$ & $\mathrm{~F}$ & $\mathrm{~F}$ & $\mathrm{~F}$ & $\mathrm{~F}$ \\
\hline$A_{2}$ & LD & $\mathrm{F}$ & LD & $\mathrm{F}$ & LD & $\mathrm{F}$ & LD \\
\hline$A_{3}$ & LD & $\mathrm{F}$ & LD & $\mathrm{F}$ & LD & LD & LD \\
\hline$A_{4}$ & $\mathrm{~F}$ & $\mathrm{~F}$ & $\mathrm{~F}$ & $\mathrm{~F}$ & $\mathrm{~F}$ & LD & LD \\
\hline$A_{5}$ & LD & $\mathrm{F}$ & LD & $\mathrm{F}$ & LD & $\mathrm{F}$ & LD \\
\hline
\end{tabular}



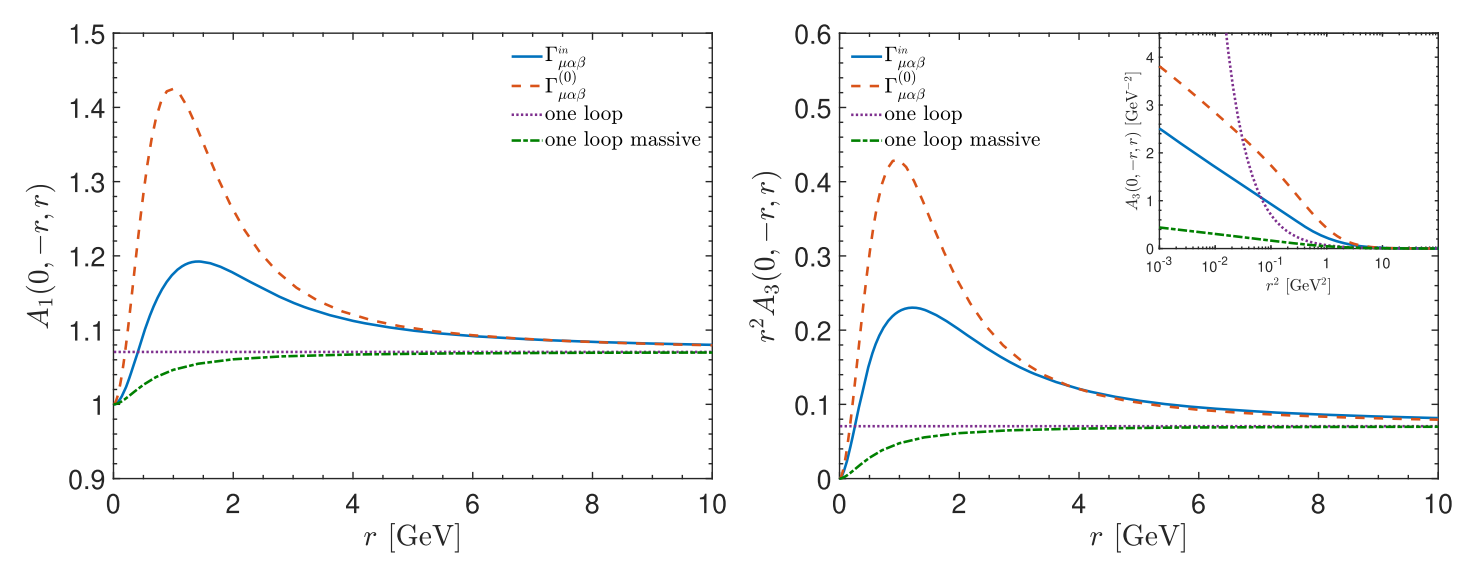

FIG. 9. (Soft antighost) Left: Comparison of the $A_{1}(0,-r, r)$ computed using $\Gamma_{\mu \alpha \beta}^{\text {in }}$ (blue continuous line) and $\Gamma_{\mu \alpha \beta}^{(0)}($ red dashed line) in the soft antighost kinematics. The massless (purple dotted line) and the massive (green dash-dotted line) one-loop perturbative results are given by Eqs. (A9) and (A10), respectively. Right: Same comparison for the dimensionless combination $r^{2} A_{3}(0,-r, r)$. In the inset we show the corresponding logarithmic infrared divergence of the $A_{3}(0,-r, r)$ and the linear divergence of the massless one-loop case.

the nonperturbative calculation with $\Gamma_{\mu \alpha \beta}^{(0)}$ display the same qualitative behavior; of course, the precise rates of each divergence are different. As can be seen in Table I, the infrared divergence found in $A_{3}$ is due to both the massless ghost entering in the diagram $\left(d_{1}\right)$ and the unprotected logarithm present in the $\Gamma_{\mu \alpha \beta}^{\text {in }}$ of graph $\left(d_{2}\right)$.

(iii) The totally symmetric limit, defined in Eq. (4.8).

In Fig. 10 we show the behavior of the $A_{i}(Q)$ in this configuration; note that in this configuration all form factors are accessible.

We clearly see that the $A_{i}$ obtained with either vertex display a sizable deviation from their tree-level value in the region of $Q \approx 1-2 \mathrm{GeV}$, while for large values of $Q$ they recover the ultraviolet behavior expected from one-loop perturbation theory, given by Eqs. (A12). Interestingly enough, except for $Q^{2} A_{5}(Q)$, the use of $\Gamma_{\mu \alpha \beta}^{\text {in }}$ yields $A_{i}$ that are more suppressed.

Moreover, one can notice that $A_{4}$ and $A_{5}$, whose forms were not presented for the previous configurations, also display a logarithmic divergence in the infrared (see the insets of Fig. 10). In the case of $A_{4}$, the divergence is exclusively associated with the unprotected logarithm present in the $\Gamma_{\mu \alpha \beta}^{\text {in }}$ used in $\left(d_{2}\right)$, while the diagram responsible for the logarithm divergence of $A_{5}$ is $\left(d_{1}\right)$ (see Table I). Notice that, except for $A_{4}(Q)$, both the analytic one-loop massive results and the nonperturbative calculation with $\Gamma_{\mu \alpha \beta}^{(0)}$ reproduce the general pattern found when one uses $\Gamma_{\mu \alpha \beta}^{\text {in }}$. More specifically, these cases capture whether the divergence is positive or negative and the finiteness of $A_{1}$. In the case of $A_{4}$, the impact of $\Gamma_{\mu \alpha \beta}^{\text {in }}$ is rather pronounced, and it causes a negative logarithmic divergence.

Table I provides an overview of our main findings, specifying the different origins of the infrared logarithmic divergences found in the form factors $A_{2}, A_{3}, A_{4}$, and $A_{5}$ in the three cases analyzed.

\section{THE CONSTRAINT FROM THE STI}

The next item of our analysis is dedicated to the STIderived constraint of Eq. (2.14). The way this particular constraint becomes relevant for our considerations is twofold. First, a considerable degree of hindsight gained from this equation has already been used in Sec. IV, in order to optimize the ultraviolet features of the input functions. Second, as we will see below, the amount by which the calculated value for $\mathcal{R}$ deviates from unity favors the use of dressed rather than bare vertices in the graphs $\left(d_{1}\right)_{\nu \mu}$ and $\left(d_{2}\right)_{\nu \mu}$.

With respect to the first point, note that the relation of Eq. (2.14), being a direct consequence of the Becchi-RouetStora-Tyutin symmetry, is satisfied exactly at any fixed order calculation in perturbation theory. However, in general, our truncation procedure does not reduce itself to a fixed order perturbative result, for any limit of the kinematic parameters. This happens because certain of the (higher order) terms, generated after the integration of all ingredients, ought to cancel/combine with contributions stemming from two- and higher-loop dressed diagrams of $H_{\nu \mu}$, which, evidently, have been omitted from the outset. The resulting mismatches, in turn, affect unequally the different kinematic configurations entering in $\mathcal{R}$, thus distorting the subtle balance that enforces Eq. (2.14).

A concrete manifestation of the underlying imbalances occurs when one uses input propagators and vertices containing perturbative information (e.g., are of the general form $\left.1+c \alpha_{s} \log q^{2} / \mu^{2}\right)$. Since one may not intervene in the actual numerical evaluation and discard "by hand" terms of $\mathcal{O}\left(\alpha_{s}^{2}\right)$ and higher, the final answer contains a certain amount of unbalanced contributions. The clearest manifestation of this effect occurs when evaluating $\mathcal{R}$ for asymptotically large momenta: contrary to what one might expect, the "tails" of $\mathcal{R}$ deviate markedly from unity; in fact, the deviation increases as the momenta grow. 

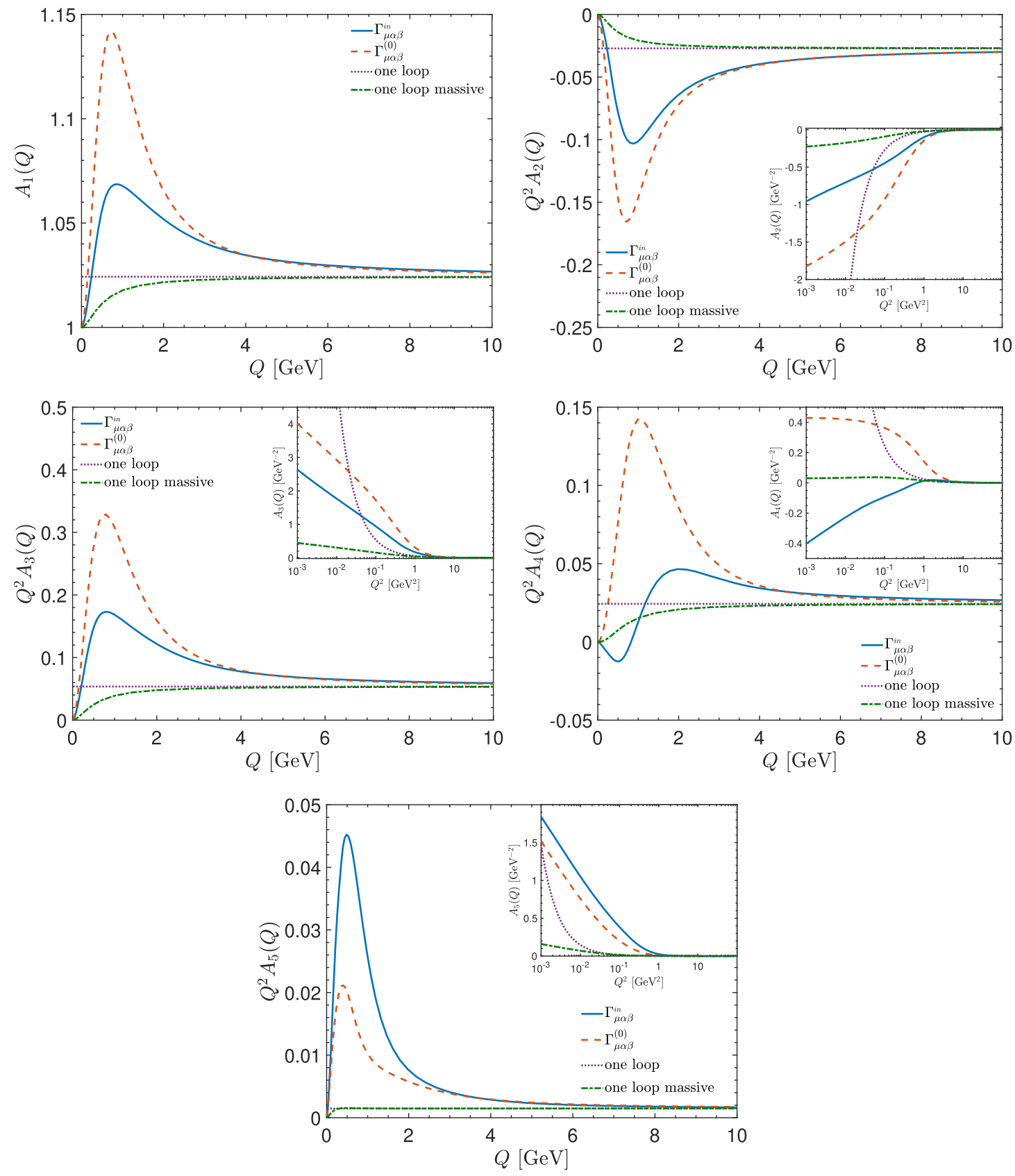

FIG. 10. (Totally symmetric) The dimensionless combinations of the form factors $A_{i}(Q)$ in the totally symmetric configuration. The $A_{i}(Q)$ are computed using $\Gamma_{\mu \alpha \beta}^{\text {in }}$ given by Eq. (4.5) (blue continuous line) and $\Gamma_{\mu \alpha \beta}^{(0)}$ of Eq. (4.7) (red dashed line). The one-loop results are given in Eqs. (A12) (purple dotted line) while the infrared limits of the one-loop massive case are expressed by Eq. (A16). In the inset we show the corresponding logarithmic divergences of the $A_{i}(Q)$ and the linear divergence of the massless one-loop result.

The use of input functions that tend to their tree-level values ameliorates the situation substantially, because, in this way, the $A_{i}$ computed display at least their correct oneloop behavior. This improvement, in turn, must be combined with a judicious choice for the $F(p)$ and $F(r)$ appearing explicitly in $\mathcal{R}$ [see Eq. (2.13)]; in particular, the function used must display asymptotically the logarithmic behavior dictated by one-loop perturbation theory. Specifically, we use the standard fit [31]

$$
\begin{aligned}
F^{-1}(q)= & 1+\frac{9 C_{\mathrm{A}} \alpha_{s}}{48 \pi}\left[1+D \exp \left(-\rho_{4} q^{2}\right)\right] \\
& \times \ln \left(\frac{q^{2}+\rho_{3} M^{2}(q)}{\mu^{2}}\right)
\end{aligned}
$$

where 

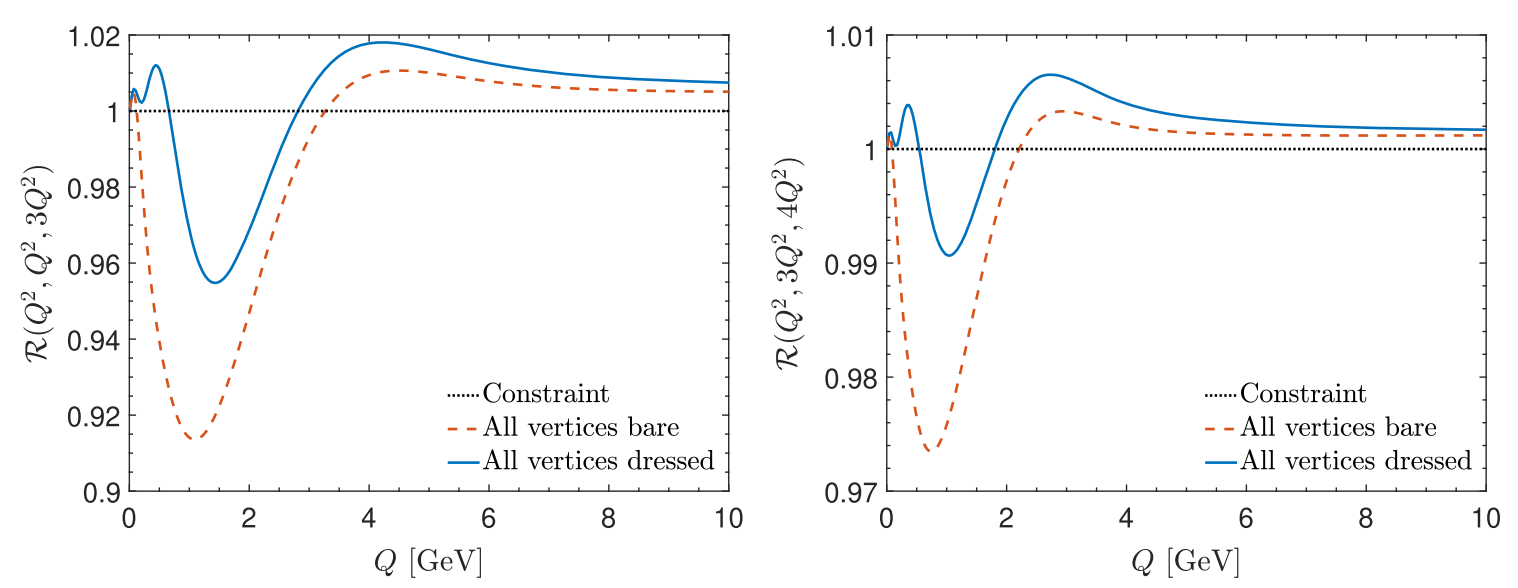

FIG. 11. The ratio $\mathcal{R}\left(q^{2}, p^{2}, r^{2}\right)$, defined in Eq. (2.13), evaluated in two different kinematic limits: (i) $p^{2}=q^{2}=Q^{2}$ and $r^{2}=3 Q^{2}$ (left panel) and (ii) $q^{2}=Q^{2}, p^{2}=3 Q^{2}$, and $r^{2}=4 Q^{2}$ (right panel). The blue continuous curve represents the case where the $A_{i}$ are computed using all vertices dressed, whereas the red dashed one is obtained when we employ bare vertices. The black dotted line represents the exact value $\mathcal{R}\left(q^{2}, p^{2}, r^{2}\right)=1$, imposed by the STI.

$$
M^{2}(q)=\frac{m_{1}^{2}}{1+q^{2} / \rho_{2}^{2}}
$$

with $\quad m_{1}^{2}=0.16 \mathrm{GeV}^{2}, \quad \rho_{2}^{2}=0.69 \mathrm{GeV}^{2}, \quad \rho_{3}=0.89$, $\rho_{4}=0.12 \mathrm{GeV}^{-2}, D=2.36$, and $\mu=4.3 \mathrm{GeV}$.

Then, after these adjustments, the tails of $\mathcal{R}$ display only a minuscule deviation from unity, which decreases slowly as the momenta increase.

We next turn to the second point and consider what the STI constraint suggests regarding the vertices used in the calculation.

Clearly, for any kinematic configuration where $|p|=|r|$, the numerator and the denominator of Eq. (2.13) become equal, and Eq. (2.14) is trivially satisfied. In particular, this is precisely what happens in the "soft antighost" and totally symmetric limits presented in the previous subsection.

Let us then consider two different kinematic limits, for which Eq. (2.14) is not trivially fulfilled. Specifically, we compute $\mathcal{R}$ for two particular kinematic configurations, shown in Fig. 11: (i) on the left panel we present $\mathcal{R}$ when $p^{2}=q^{2}=Q^{2}$ and $r^{2}=3 Q^{2}$, or, equivalently, $\theta=\pi / 3$; we denote the corresponding quantity by $\mathcal{R}\left(Q^{2}, Q^{2}, 3 Q^{2}\right)$ [alternatively, $\mathcal{R}\left(Q^{2}, Q^{2}, \pi / 3\right)$ ]; (ii) on the right panel, we present the case $q^{2}=Q^{2}, p^{2}=3 Q^{2}$, and $r^{2}=4 Q^{2}$, which corresponds to $\theta=\pi / 2$; we denote the result by $\mathcal{R}\left(Q^{2}, 3 Q^{2}, 4 Q^{2}\right)$. As a reference, in Fig. 11 we plot the ideal result $\mathcal{R}\left(q^{2}, p^{2}, r^{2}\right)=1$ (black dotted line), corresponding to the STI constraint of Eq. (2.14).

Notice that in both cases we evaluate $\mathcal{R}\left(q^{2}, p^{2}, r^{2}\right)$ using two different approximations: (a) the $A_{i}$ are computed using tree-level expressions for the full vertices appearing in the diagrammatic representation of $H^{\nu \mu}$ (red dashed curves), and (b) the $A_{i}$ are computed with all vertices dressed [see Eq. (3.2)], using the Ansätze discussed in Sec. III (blue continuous curves).
In the left panel, one clearly observes that the maximum deviation from unity occurs for $q$ in the range $1.0-1.5 \mathrm{GeV}$, being around $9 \%$ when tree-level vertices are used, and dropping below $5 \%$ when all vertices are dressed. Then, in the perturbative region, for values of $q \geq 5 \mathrm{GeV}$ the deviations in both cases are smaller than $2 \%$.

In the kinematic configuration presented in the right panel, we notice that the deviations are milder. Specifically, the maximum deviation appears in the momentum range $0.8-1.1 \mathrm{GeV}$ and is less than $3 \%$ when bare vertices are used, dropping to less than $1 \%$ for dressed vertices. In the ultraviolet the deviation from unity is of the order of $0.1 \%$.

The difference between the ideal and computed values of $\mathcal{R}$ may be quantified by means of a $\chi^{2}$ test. The test was implemented using the 80 points of our logarithmic grid, defined in the entire range of momenta, i.e., $\left[5 \times 10^{-5} \mathrm{GeV}^{2}, 5 \times 10^{3} \mathrm{GeV}^{2}\right]$. Note that the logarithmically spaced grid furnishes more weight to the nonperturbative region because it has a higher concentration of points in the infrared.

For the case of the bare vertices we obtain $\chi^{2}=0.057$ (left panel) and $\chi^{2}=0.004$ (right panel), whereas for the dressed case one has $\chi^{2}=0.021$ (left panel) and $\chi^{2}=$ 0.001 (right panel); evidently, these results favor the truncation scheme where all vertices are dressed.

Alternatively, one may also use as an indicator of the similarity of the two curves the integral over the absolute value of the difference of them. More specifically, we have evaluated the following integral:

$$
\mathcal{I}_{a b}=\int_{\Lambda_{\mathrm{IR}}}^{\Lambda_{\mathrm{UV}}}\left|\mathcal{R}\left(Q^{2}, a Q^{2}, b Q^{2}\right)-1\right| d Q,
$$

where the values of $a$ and $b$ are fixed by the choice of momenta in each configuration; for the two examples considered in Fig. 11 we have $(a=1, b=3)$ and $(a=3, b=4)$, respectively. For the case of the bare 
vertices we find $\mathcal{I}_{13}=0.32$ and $\mathcal{I}_{34}=0.076$, whereas for the dressed case one has $\mathcal{I}_{13}=0.29$ and $\mathcal{I}_{34}=0.068$. Evidently, this second indicator displays a slight preference for the truncation scheme where all vertices are dressed, but is considerably less compelling compared to the $\chi^{2}$ case.

\section{RESULTS FOR THE GHOST-GLUON VERTEX}

As a direct application of the results obtained for the $A_{i}$ in the previous section, we now turn our attention to the determination of the form factors of the ghost-gluon vertex, for arbitrary Euclidean momenta. To that end, we use the exact expressions given by Eq. (2.8), which was derived from Eq. (2.4) [88].

In Figs. 12 and 13 we show, respectively, the form factors $B_{1}$ and $B_{2}$ as functions of $q^{2}, p^{2}$, and $\theta$. In order to appreciate their angular dependence, we present two representative cases: $\theta=0$ and $\theta=\pi$. As we can see, the angular dependence of $B_{1}$ is relatively weak, whereas $B_{2}$ is clearly more sensitive to changes in $\theta$. Note also that both form factors approach their one-loop perturbative
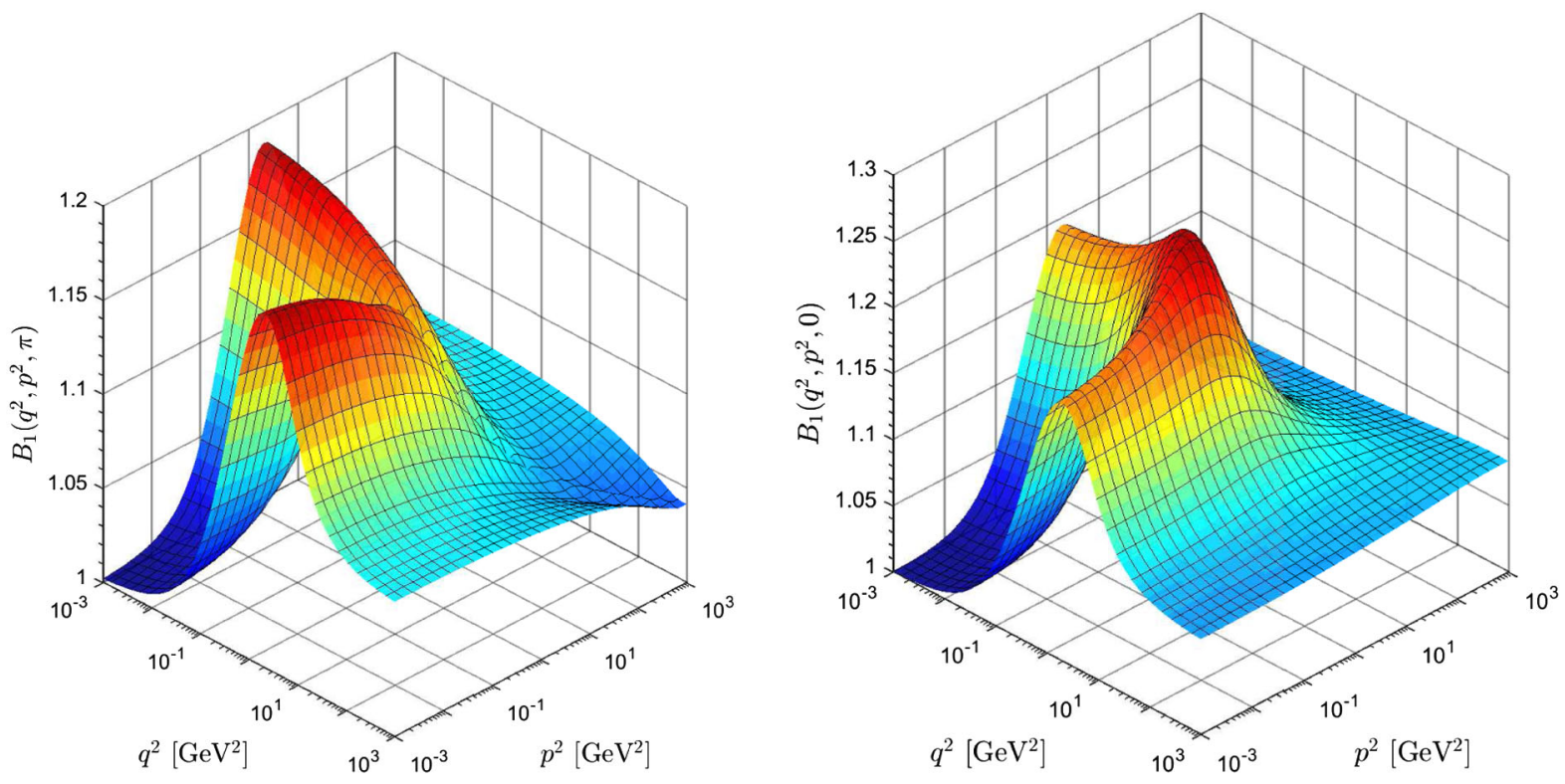

FIG. 12. Form factor $B_{1}\left(q^{2}, p^{2}, \theta\right)$ of the ghost-gluon vertex, for $\theta=0$ (left panel) and $\theta=\pi$ (right panel).
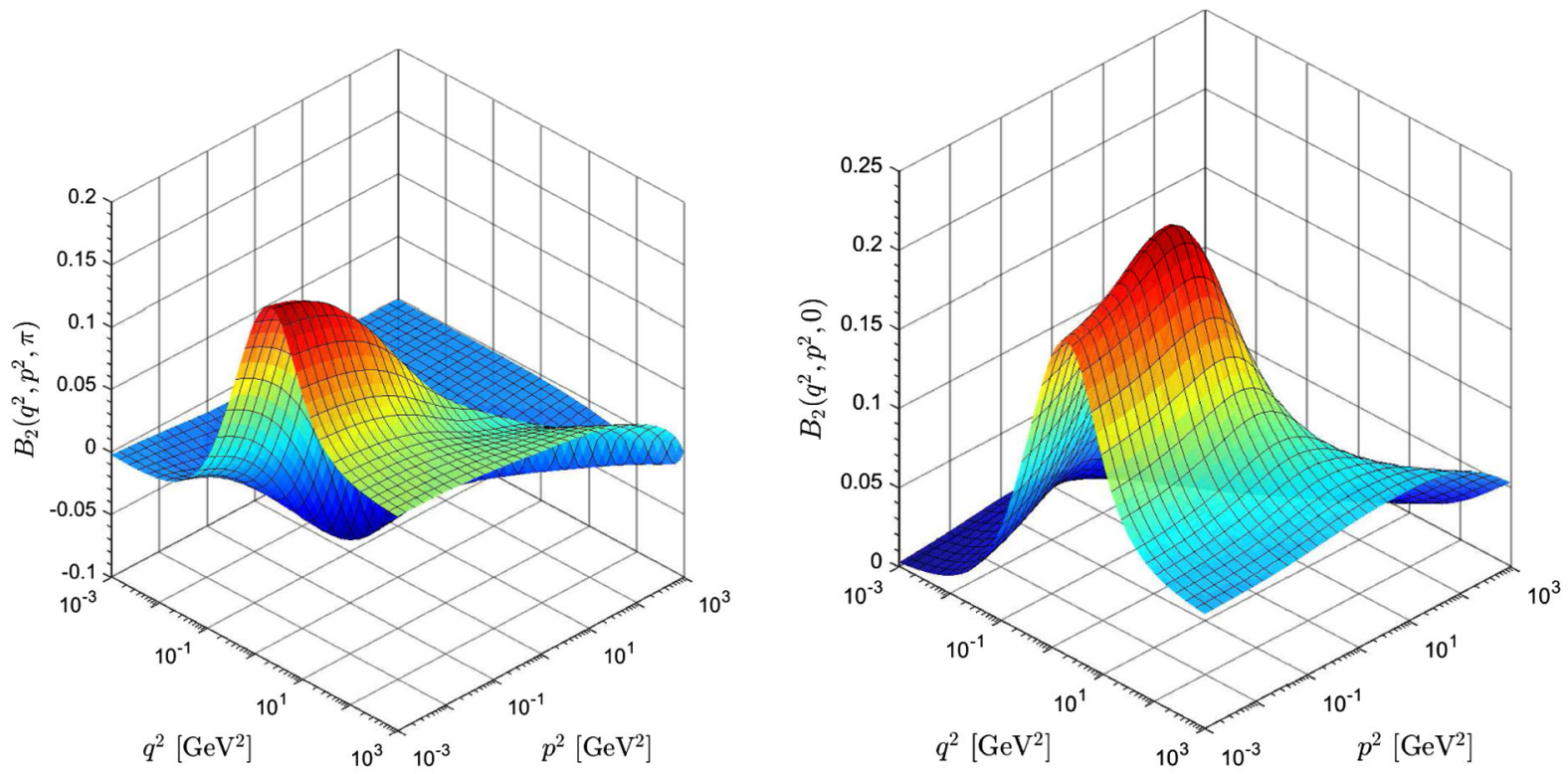

FIG. 13. Form factor $B_{2}\left(q^{2}, p^{2}, \theta\right)$ of the ghost-gluon vertex, for $\theta=0$ (left panel) and $\theta=\pi$ (right panel). 

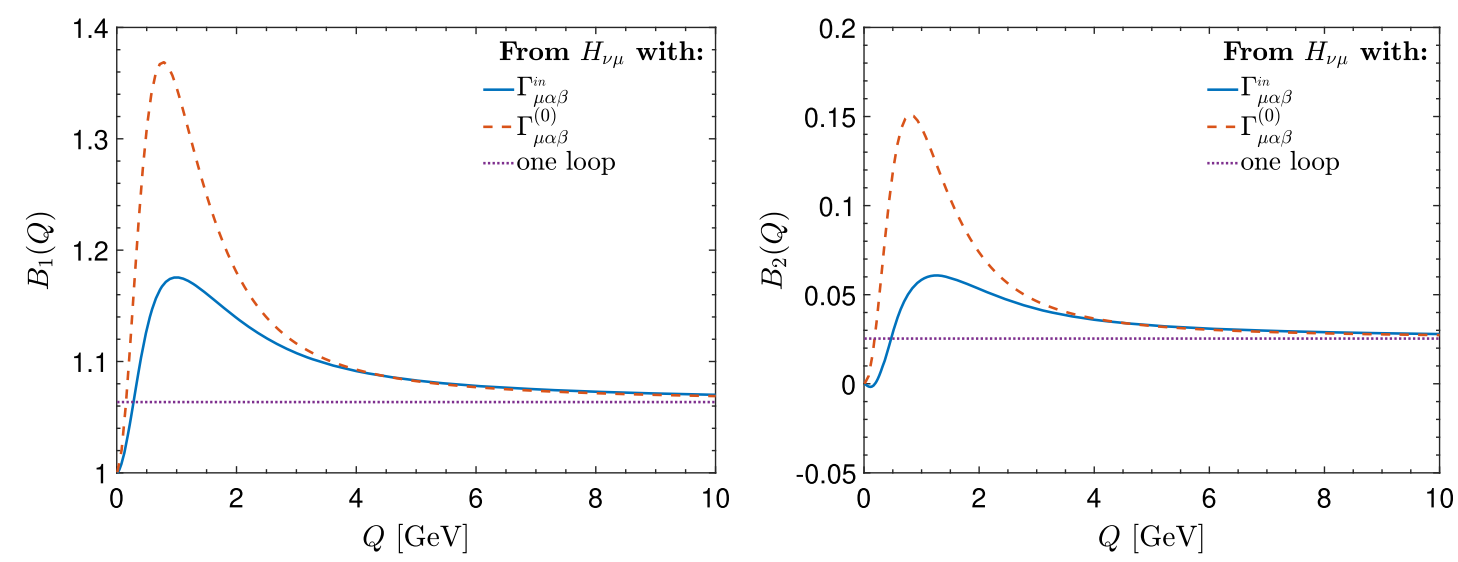

FIG. 14. $\quad B_{1}(Q)$ (left panel) and $B_{2}(Q)$ (right panel) in the totally symmetric configuration obtained when the $A_{i}$ entering in Eq. (2.8) are computed using the three-gluon vertex dressed given by Eq. (4.5) (blue continuous line) or at tree level given by Eq. (4.7) (red dashed line). The one-loop results for $B_{1}(Q)$ and $B_{2}(Q)$ (purple dotted line) may be directly obtained combining Eqs. (2.8) and (A12).

behavior $^{10}$ whenever one of the ghost $(p)$ or antighost $(q)$ momenta becomes large.

In addition, for $p^{2}=q^{2}=0$ they revert to their treelevel values, due to the fact that the one-loop dressed contributions to $H_{\nu \mu}$ vanish at the origin. Moreover, we may visually verify that $B_{1}\left(q^{2}, p^{2}, \theta\right)$ is symmetric under the exchange $q^{2} \leftrightarrow p^{2}$, for any $\theta$, as required by the ghostantighost symmetry.

It is clear that $B_{1}$ and $B_{2}$ will depend through the $A_{i}$ on our choice for $\Gamma_{\mu \alpha \beta}$. In order to study this effect, we employ the results presented in Sec. VB, where the $A_{i}$ were computed using as input for $\Gamma_{\mu \alpha \beta}$ either the $\Gamma_{\mu \alpha \beta}^{(0)}$ of Eq. (4.7) or the $\Gamma_{\mu \alpha \beta}^{\mathrm{in}}$ of Eq. (4.5). In Fig. 14 we show the results of this study for $B_{1}(Q)$ and $B_{2}(Q)$ in the totally symmetric configuration. Clearly, when the three-gluon vertex is dressed, the results for $B_{1}$ and $B_{2}$ are systematically suppressed. Notice that the relative difference is more pronounced in the intermediate region of momenta, given that in the deep infrared we must have $B_{1}(0,0,0)=1$ and $B_{2}(0,0,0)=0$, while in the ultraviolet $B_{1}$ and $B_{2}$ should recover the expected perturbative behavior. In particular, around the region of $0.9-1.1 \mathrm{GeV}$, the deviations of $B_{1}$ and $B_{2}$ from their tree-level values are approximately 2-2.5 times larger when $\Gamma_{\mu \alpha \beta}^{(0)}$ is used.

Next, in Fig. 15, we compare our results for $B_{1}$ in the soft gluon configuration with those obtained in earlier works [48-50]; this configuration is the most widely explored in the literature, being the only one simulated on lattice for

\footnotetext{
${ }^{10}$ Notice that the one-loop behavior for $B_{1}$ in the soft ghost, soft gluon, and totally symmetric configurations deviates slightly from 1, being 1.07, 1.04, and 1.06, respectively. The corresponding relative errors between our nonperturbative computation and the expected one-loop behavior are smaller than $1 \%$ for momenta higher than $8 \mathrm{GeV}$, in the three kinematic configurations mentioned-see e.g., Fig. 14.
}

$\mathrm{SU}(3)[63,64]$. The green dash-dotted curve represents the results for $B_{1}(q,-q, 0)$, obtained from the approach developed in [48], based on the infrared completion of expressions derived using operator product expansion techniques. In the case of [49], $B_{1}$ was determined in general kinematics, using a system of coupled SDEs, while in [50] the $B_{1}$ was determined exclusively in the soft gluon configuration. It is interesting to notice that all analytical studies display the characteristic peak and converge to unity at the origin. Moreover, all of them are in qualitative agreement with the lattice data (note, however, that the error bars are quite sizable).

Finally, in Fig. 16, we illustrate the impact that the full structure of $B_{1}\left(q^{2}, p^{2}, \theta\right)$ has on the SDE of the ghost dressing function. To that end, we explore two scenarios: (i) we couple the entire momenta dependence of $B_{1}$ to the SDE for $F(q)$, carrying out the additional angular integration [see Eq. (2.14) of [71] ], and (ii) we fix its momentum

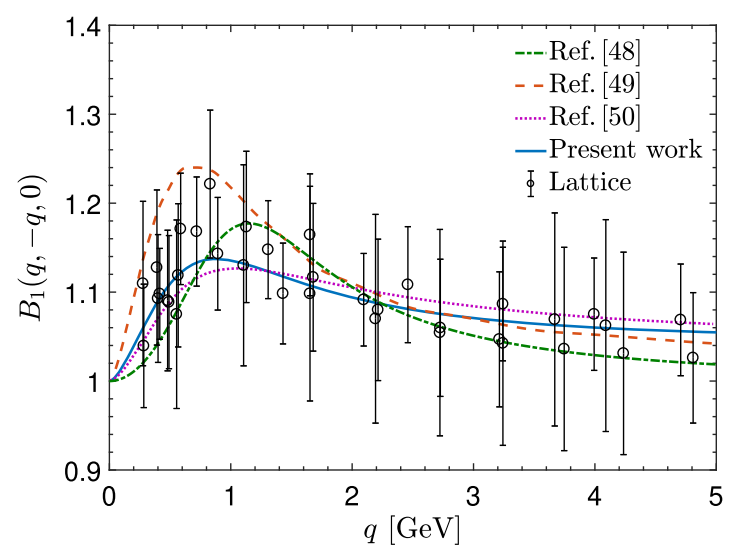

FIG. 15. Our numerical result for $B_{1}(q,-q, 0)$ (blue continuous line) compared with the results of [48] (green dash-dotted line), [49] (red dashed line), and [50] (magenta dotted line). The lattice data (circles) are from $[63,64]$. 

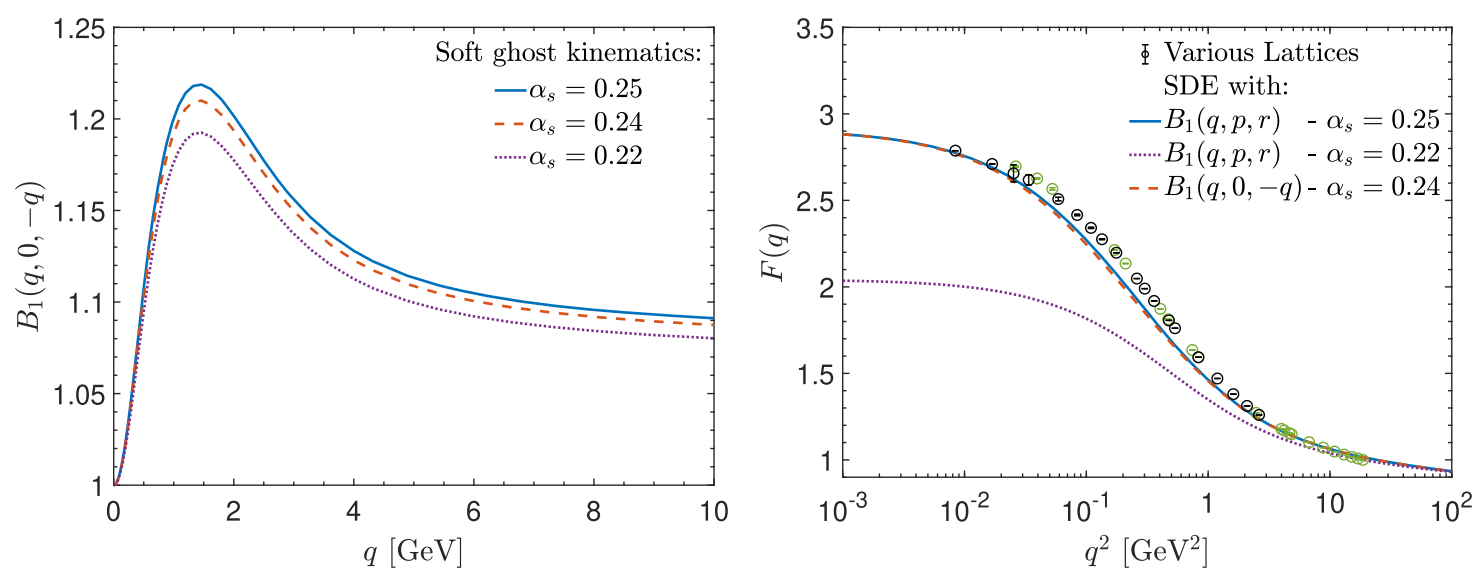

FIG. 16. The $B_{1}(q, 0,-q)$ computed using three different values of $\alpha_{s}$ (left panel). The $F(q)$ obtained by substituting into the ghost SDE: (a) the full $B_{1}(q, p, r)$ with $\alpha_{s}=0.25$ (blue continuous line), (b) the full $B_{1}(q, p, r)$ with $\alpha_{s}=0.22$ (purple dotted line), and (c) the soft gluon limit $B_{1}(q, 0,-q)$ with $\alpha_{s}=0.24$ (red dashed line). The lattice data are from [54,55] (right panel).

dependence to the soft ghost configuration [47,71]. We observe that with mild adjustments to the value of $\alpha_{s}$, both scenarios reproduce the standard lattice results of [54] rather accurately; in particular, while for case (i) $\alpha_{s}=0.25$, for (ii) we obtain $\alpha_{s}=0.24$.

The reason for this small difference in the values of $\alpha_{s}$ can easily be understood. As mentioned in Sec. V B, in the region of momenta of about 2 to 3 times the QCD mass scale, the soft ghost configuration maximizes the deviation from the tree-level value. Therefore, when we approximate the entire momentum dependence of $\Gamma^{\mu}$ just by this configuration (instead of integrating over all of them), we slightly overestimate the contribution of the ghost-gluon vertex to the ghost SDE.

It is also interesting to notice that, although the impact of changing the value of $\alpha_{s}$ is rather mild at the level of $B_{1}(q, 0,-q)$, it is rather pronounced when $F(q)$ is computed, as clearly seen in Fig. 16. More specifically, the relative difference between the $B_{1}(q, 0,-q)$ computed with $\alpha_{s}=0.25$ and that computed with $\alpha_{s}=0.22$ is less than $3 \%$ around the region of the peaks. Instead, in the case of $F(q)$, the relative difference between the corresponding curves increases to $30 \%$ in the deep infrared; this, in turn, may be traced back to the known high sensitivity of the ghost SDE on the value of $\alpha_{s}$.

\section{CONCLUSIONS}

We have presented a detailed nonperturbative study of the form factors, $A_{i}$, comprising the ghost-gluon kernel, $H_{\nu \mu}$, using the one-loop dressed approximation of its dynamical equation, for general Euclidean momenta. The results obtained have been presented in $3 \mathrm{D}$ plots, and certain slices, corresponding to special kinematic limits, have been singled out and inspected in detail. The $A_{i}$ obtained have been subsequently used for the determination of the two form factors, $B_{1}$ and $B_{2}$, of the ghost-gluon vertex.
The ingredients entering in the calculations are the gluon and ghost propagators, and the vertices $\Gamma_{\alpha \mu \nu}$ and $\Gamma_{\mu}$. Given that the $H_{\nu \mu}$ itself is intimately connected to both these vertices, a strictly self-consistent treatment would require one to couple the dynamical equation governing $H_{\nu \mu}$ to the equations relating it to both $\Gamma_{\alpha \mu \nu}$ and $\Gamma_{\mu}$, and proceed to the solution of the entire coupled system. Instead, we have treated the problem at hand by employing simplified versions of these vertices, whose use in recent studies $[93,108]$ yielded satisfactory results. Moreover, as has been explained in detail, there exists a subtle interplay between the truncation of the equations employed, the ultraviolet behavior of the ingredients used for their evaluation, and the accuracy with which the resulting $A_{i}$ satisfy the STI constraint of Eq. (2.14). Note in particular that while our input expressions for the two-point functions are in excellent agreement with the lattice data of [54] for infrared and intermediate momenta, their ultraviolet tails have been adjusted to their tree-level values.

We have paid particular attention to the impact that the structure of $\Gamma_{\alpha \mu \nu}$ may have on the results. All our findings indicate that the use of a dressed $\Gamma_{\alpha \mu \nu}$, corresponding to the so-called "minimal BC solution," $\Gamma_{\mu \alpha \beta}^{\mathrm{in}}$, induces an appreciable suppression with respect to the results obtained by merely resorting to $\Gamma_{\alpha \mu \nu}^{(0)}$. This happens because the form factor $X_{1}^{\text {in }}$ is itself suppressed in the infrared, due to the form of the functions $J(q)$ that enter its definition [see Eq. (4.6)]. This special feature of the three-gluon vertex, in turn, appears to be favored by the STI-derived constraint, in the sense that the results obtained with $\Gamma_{\mu \alpha \beta}^{\text {in }}$ are considerably closer to unity (see Fig. 11).

The information obtained on the structure of the ghostgluon kernel opens the way toward the systematic nonperturbative construction of the 10 form factors comprising the longitudinal part of the three-gluon vertex, using the BC construction [87] as a starting point. The detailed 
knowledge of these form factors, in turn, may have considerable impact on the study of the dynamical formation of gluon dominated bound states, such as glueballs and hybrids (see, e.g., [109], and references therein). We hope to be able to present results on this topic in the near future.

\section{ACKNOWLEDGMENTS}

The research of J. P. is supported by the Spanish MEyC under Grants No. FPA2017-84543-P and No. SEV-20140398, and Generalitat Valenciana under Grant Prometeo II/ 2014/066. The work of A. C. A and M. N. F. is supported by the Brazilian National Council for Scientific and Technological Development (CNPq) under Grants No. 305815/2015 and No. 142226/2016-5, respectively. A. C. A and C. T. F. also acknowledge the financial support from São Paulo Research Foundation (FAPESP) through Projects No. 2017/07595-0, No. 2017/05685-2, No. 2016/ 11894-0, and No. 2018/09684-3. This study was financed in part by the Coordenação de Aperfeićoamento de Pessoal de Nível Superior_Brasil (CAPES)—Finance Code 001 (M. N. F.). This research was performed using the Feynman Cluster of the John David Rogers Computation Center (CCJDR) in the Institute of Physics "Gleb Wataghin," University of Campinas.

\section{APPENDIX A: ONE-LOOP RESULTS FOR SPECIAL KINEMATIC CONFIGURATIONS}

In this Appendix we present the one-loop results for the various $A_{i}$ in the three special kinematic configurations considered in Sec. VB $[94,110]$. In addition, for two kinematic limits, we also show the corresponding results for the one-loop massive case, where the gluon propagator is endowed with a hard mass $m$.

The one-loop calculations are performed analytically; the starting expressions may be obtained from the $\left(d_{1}\right)_{\nu \mu}$ and $\left(d_{2}\right)_{\nu \mu}$ of Eq. (3.2) by replacing the dressed quantities by their tree-level counterparts, i.e., $D(q)=i / q^{2}, \mathcal{V}_{1}=$ $\mathcal{V}_{2}=1$, and $J(q)=1$. In the case of the gluon propagator, the corresponding tree-level expressions used are either $\Delta(q)=1 / q^{2}$ (for the "conventional" one-loop calculation) or $\Delta^{-1}(q)=q^{2}-m^{2}$ (for the one-loop massive calculation).

In addition, we employ dimensional regularization, in which case the measure of Eq. (3.2) assumes the form

$$
\int_{\ell} \rightarrow \frac{\mu^{\epsilon}}{(2 \pi)^{d}} \int \mathrm{d}^{d} \ell
$$

where $d=4-\epsilon$, and $\mu$ is the 't Hooft mass scale.

Implementing the substitutions mentioned above, we obtain for the conventional one-loop case

$$
\begin{aligned}
& \left(d_{1}\right)_{\nu \mu}^{(1)}=\frac{i g^{2} C_{\mathrm{A}}}{2} \int_{\ell} \frac{(\ell-q)_{\mu}}{\ell^{2}(\ell+p)^{2}(\ell-q)^{2}}\left[p_{\nu}-\ell_{\nu} \frac{(p \cdot \ell)}{\ell^{2}}\right], \\
& \left(d_{2}\right)_{\nu \mu}^{(1)}=\frac{i g^{2} C_{\mathrm{A}}}{2} \int_{\ell} \frac{\Gamma_{\mu \sigma \alpha}^{(0)}}{\ell^{2}(\ell+r)^{2}(\ell-q)^{2}}\left[g_{\nu}^{\sigma}-\frac{\ell^{\sigma} \ell_{\nu}}{\ell^{2}}\right]\left[p^{\alpha}-\frac{p \cdot(\ell+r)(\ell+r)^{\alpha}}{(\ell+r)^{2}}\right],
\end{aligned}
$$

while for the one-loop massive case one has

$$
\begin{aligned}
& \left(d_{1}\right)_{\nu \mu}^{(1 \mathrm{M})}=\frac{i g^{2} C_{\mathrm{A}}}{2} \int_{\ell} \frac{(\ell-q)_{\mu}}{\left(\ell^{2}-m^{2}\right)(\ell+p)^{2}(\ell-q)^{2}}\left[p_{\nu}-\ell_{\nu} \frac{(p \cdot \ell)}{\ell^{2}}\right], \\
& \left(d_{2}\right)_{\nu \mu}^{(1 \mathrm{M})}=\frac{i g^{2} C_{\mathrm{A}}}{2} \int_{\ell} \frac{\Gamma_{\mu \sigma \alpha}^{(0)}}{\left(\ell^{2}-m^{2}\right)\left[(\ell+r)^{2}-m^{2}\right](\ell-q)^{2}}\left[g_{\nu}^{\sigma}-\frac{\ell^{\sigma} \ell_{\nu}}{\ell^{2}}\right]\left[p^{\alpha}-\frac{p \cdot(\ell+r)(\ell+r)^{\alpha}}{(\ell+r)^{2}}\right],
\end{aligned}
$$

where $\Gamma_{\mu \sigma \alpha}^{(0)}=(2 r+l)_{\alpha} g_{\mu \sigma}-(2 l+r)_{\mu} g_{\alpha \sigma}+(l-r)_{\sigma} g_{\mu \alpha}$.

For numerical purposes, the mass appearing in the oneloop massive calculation will be fixed $m^{2}=0.15 \mathrm{GeV}^{2}$. This value coincides with the dynamical gluon mass at zero momentum, namely the value of $\Delta^{-1}(0)$, for $\mu=4.3 \mathrm{GeV}$ [see Eqs. (4.1) and (4.3)].

Below we summarize the results (in Euclidean space) obtained after introducing the Feynman parametrization and using PACKAGE-X [103,104].

(1) Soft gluon limit: To derive this configuration, we set $r \rightarrow 0$ directly into Eqs. (2.3) and (A2). It is straightforward to see that in this limit the tensorial structure of $H_{\nu \mu}^{(1)}(q, 0)$ given by (2.3) reduces to

$$
\begin{aligned}
H_{\nu \mu}^{(1)}(q,-q, 0)= & A_{1}^{(1)}(q,-q, 0) g_{\mu \nu} \\
& +A_{2}^{(1)}(q,-q, 0) q_{\mu} q_{\nu}
\end{aligned}
$$

and the form factors become

(a) One loop:

$$
A_{1}^{(1)}(q,-q, 0)=1 ; \quad A_{2}^{(1)}(q,-q, 0)=-\frac{3 C_{\mathrm{A}} \alpha_{s}}{16 \pi q^{2}} .
$$

Then, the one-loop result for $B_{1}^{(1)}(q,-q, 0)$ may be directly obtained using Eq. (2.8). 
(b) One-loop massive:

$$
\begin{aligned}
A_{1}^{(1 \mathrm{M})}(q,-q, 0)= & 1-\frac{C_{\mathrm{A}} \alpha_{s}}{192 \pi m^{4} q^{4}}\left[\left(10 m^{8}+8 m^{6} q^{2}\right) \ln \left(\frac{m^{2}}{m^{2}+q^{2}}\right)+10 m^{6} q^{2}\right. \\
& \left.+3 m^{4} q^{4}+2 m^{2} q^{6}+\left(4 m^{2} q^{6}-2 q^{8}\right) \ln \left(\frac{q^{2}}{m^{2}+q^{2}}\right)\right] \\
A_{2}^{(1 \mathrm{M})}(q,-q, 0)= & -\frac{C_{\mathrm{A}} \alpha_{s}}{96 \pi m^{4} q^{6}}\left[20 m^{6} q^{2}+15 m^{4} q^{4}+q^{6}\left(4 q^{2}-m^{2}\right) \ln \left(\frac{q^{2}}{m^{2}}\right)\right. \\
& \left.+\left(20 m^{8}+25 m^{6} q^{2}-m^{2} q^{6}+4 q^{8}\right) \ln \left(\frac{m^{2}}{m^{2}+q^{2}}\right)+4 m^{2} q^{6}\right] .
\end{aligned}
$$

Evidently, in the limit $m \rightarrow 0$, one recovers the one-loop results given by Eq. (A5). Moreover, in the infrared limit $q \rightarrow 0$, Eq. (A6) reduces to

$$
\begin{aligned}
& \lim _{q^{2} \rightarrow 0} A_{1}^{(1 \mathrm{M})}(q,-q, 0)=1 \\
& \lim _{q^{2} \rightarrow 0} A_{2}^{(1 \mathrm{M})}(q,-q, 0)=\frac{C_{\mathrm{A}} \alpha_{s}}{576 \pi m^{2}}\left[6 \ln \left(\frac{q^{2}}{m^{2}}\right)-59\right] .
\end{aligned}
$$

Therefore, in the massive one-loop analysis, one finds that $A_{1}^{(1 \mathrm{M})}$ is infrared finite, whereas $A_{2}^{(1 \mathrm{M})}$ displays a logarithmic divergence.

(2) Soft antighost limit: This limit is obtained by setting $q=0$. The one-loop expression for $H_{\nu \mu}^{(1)}$ becomes

$$
H_{\nu \mu}^{(1)}(0,-r, r)=A_{1}^{(1)}(0,-r, r) g_{\mu \nu}+A_{3}^{(1)}(0,-r, r) r_{\mu} r_{\nu}
$$

with the two form factors given by

(a) One loop:

$$
A_{1}^{(1)}(0,-r, r)=1+\frac{11 C_{\mathrm{A}} \alpha_{s}}{32 \pi} ; \quad A_{3}^{(1)}(0,-r, r)=\frac{11 C_{\mathrm{A}} \alpha_{s}}{32 \pi r^{2}} .
$$

(b) One-loop massive:

$$
\begin{aligned}
A_{1}^{(1 \mathrm{M})}(0,-r, r)= & 1-\frac{C_{\mathrm{A}} \alpha_{s}}{192 \pi m^{6} r^{4}}\left[2 m^{8} r^{2}-23 m^{6} r^{4}+r^{6}\left(2 m^{4}-6 m^{2} r^{2}+r^{4}\right) \ln \left(\frac{r^{2}}{m^{2}}\right)\right. \\
& +\left(r^{9}-6 m^{2} r^{7}-40 m^{4} r^{5}\right) \sqrt{4 m^{2}+r^{2}} \ln \left[\frac{\left(\sqrt{4 m^{2} r^{2}+r^{4}}+r^{2}\right)}{2 m^{2}}+1\right] \\
& \left.+2\left(m^{2}+r^{2}\right)^{2}\left(m^{6}-13 m^{4} r^{2}-7 m^{2} r^{4}+r^{6}\right) \ln \left(\frac{m^{2}}{m^{2}+r^{2}}\right)\right], \\
A_{3}^{(1 \mathrm{M})}(0,-r, r)= & -\frac{C_{\mathrm{A}} \alpha_{s}}{192 \pi m^{6} r^{6}}\left[8 m^{8} r^{2}-20 m^{6} r^{4}+6 m^{4} r^{6}+r^{6}\left(8 m^{4}+r^{4}\right) \ln \left(\frac{r^{2}}{m^{2}}\right)\right. \\
& +\left(r^{9}-6 m^{2} r^{7}-40 m^{4} r^{5}\right) \sqrt{4 m^{2}+r^{2}} \ln \left[\frac{\left(\sqrt{4 m^{2} r^{2}+r^{4}}+r^{2}\right)}{2 m^{2}}+1\right] \\
& \left.+2\left(m^{2}+r^{2}\right)^{2}\left(4 m^{6}-16 m^{4} r^{2}-4 m^{2} r^{4}+r^{6}\right) \ln \left(\frac{m^{2}}{m^{2}+r^{2}}\right)\right] .
\end{aligned}
$$


Note that, when we take the limit of $m \rightarrow 0$ in the above expressions, we recover the one-loop result given in Eq. (A9). Moreover, in the limit $q \rightarrow 0$, the form factors of Eq. (A10) reduce to

$$
\begin{aligned}
\lim _{r^{2} \rightarrow 0} A_{1}^{(1 \mathrm{M})}(0,-r, r)= & 1, \\
\lim _{r^{2} \rightarrow 0} A_{3}^{(1 \mathrm{M})}(0,-r, r)= & -\frac{C_{\mathrm{A}} \alpha_{s}}{288 \pi m^{2}} \\
& \times\left[12 \ln \left(\frac{r^{2}}{m^{2}}\right)-31\right],
\end{aligned}
$$

where we confirm that $A_{1}^{(1 \mathrm{M})}$ is infrared finite, while $A_{3}^{(1 \mathrm{M})}$ is logarithmically divergent.

(3) Symmetric configuration: This kinematic limit is defined in (4.8); in this case all form factors survive, and are given by

(a) One loop:

$$
\begin{aligned}
& A_{1}^{(1)}(Q)=1+\frac{C_{\mathrm{A}} \alpha_{s}}{96 \pi}[9+\mathrm{I}], \\
& A_{2}^{(1)}(Q)=-\frac{C_{\mathrm{A}} \alpha_{s}}{48 \pi Q^{2}}[4+\mathrm{I}], \\
& A_{3}^{(1)}(Q)=\frac{C_{\mathrm{A}} \alpha_{s}}{96 \pi Q^{2}}[4+9 \mathrm{I}], \\
& A_{4}^{(1)}(Q)=\frac{C_{\mathrm{A}} \alpha_{s}}{48 \pi Q^{2}}[1+2 \mathrm{I}], \\
& A_{5}^{(1)}(Q)=\frac{C_{\mathrm{A}} \alpha_{s}}{48 \pi Q^{2}}[-2+\mathrm{I}],
\end{aligned}
$$

where I is a constant [110] defined as

$$
\mathrm{I}=\frac{1}{3}\left[\psi_{1}\left(\frac{1}{3}\right)-\psi_{1}\left(\frac{2}{3}\right)\right]=2.34391
$$

with $\psi_{1}(z)$ being the "trigamma function," expressed in terms of the standard $\Gamma(z)$ function as

$$
\psi_{1}(z)=\frac{d^{2}}{d z^{2}} \ln [\Gamma(z)]
$$

and it has the following special values:

$$
\psi_{1}\left(\frac{1}{3}\right)=10.0956, \quad \psi_{1}\left(\frac{2}{3}\right)=3.06388 .
$$

(b) One-loop massive:

The resulting expressions for the one-loop massive case are rather lengthy and will not be reported here. However, their infrared limits as $q \rightarrow 0$ are given by

$$
\begin{aligned}
& \lim _{Q^{2} \rightarrow 0} A_{1}^{(1 \mathrm{M})}(Q)=1, \\
& \lim _{Q^{2} \rightarrow 0} A_{2}^{(1 \mathrm{M})}(Q)=\frac{C_{\mathrm{A}} \alpha_{s}}{576 \pi m^{2}}\left[6 \ln \left(\frac{Q^{2}}{m^{2}}\right)-65\right], \\
& \lim _{Q^{2} \rightarrow 0} A_{3}^{(1 \mathrm{M})}(Q)=-\frac{C_{\mathrm{A}} \alpha_{s}}{144 \pi m^{2}}\left[6 \ln \left(\frac{Q^{2}}{m^{2}}\right)-23+3 \mathrm{I}\right], \\
& \lim _{Q^{2} \rightarrow 0} A_{4}^{(1 \mathrm{M})}(Q)=\frac{C_{\mathrm{A}} \alpha_{s}}{48 \pi m^{2}}, \\
& \lim _{Q^{2} \rightarrow 0} A_{5}^{(1 \mathrm{M})}(Q)=-\frac{C_{\mathrm{A}} \alpha_{s}}{192 \pi m^{2}}\left[6 \ln \left(\frac{Q^{2}}{m^{2}}\right)-1+4 \mathrm{I}\right] .
\end{aligned}
$$

Therefore, for the one-loop massive case, one finds that $A_{1}^{(1 \mathrm{M})}$ and $A_{4}^{(1 \mathrm{M})}$ are infrared finite, while $A_{2}^{(1 \mathrm{M})}, A_{3}^{(1 \mathrm{M})}$, and $A_{5}^{(1 \mathrm{M})}$ are logarithmically divergent.

\section{APPENDIX B: EXPLICIT EXPRESSIONS FOR THE $\boldsymbol{A}_{i}$}

We write the $A_{i}$ as the sum of their tree-level value and the contributions from $\left(d_{1}\right)_{\nu \mu}$ and $\left(d_{2}\right)_{\nu \mu}$, so that $A_{i}=$ $A_{i}^{(0)}+A_{i}^{\left(d_{1}\right)}+A_{i}^{\left(d_{2}\right)}$, where $A_{1}^{(0)}=1$ and $A_{i}^{(0)}=0$ for $i=2,3,4,5$.

We then introduce new kinematic variables $s=q-\ell$, $t=-\ell-p, u=-p-q$, and $v=-\ell+p+q$, the inner products $a_{1}=\ell \cdot p, a_{2}=\ell \cdot q$, and $a_{3}=p \cdot q$, together with the combinations

$$
\begin{aligned}
& T_{1}=h_{p q}+3\left(p^{2}+a_{3}\right)^{2}, \quad T_{2}=h_{p q}+3\left(q^{2}+a_{3}\right)^{2}, \\
& T_{3}=-p^{2} q^{2}+p^{4}-2 a_{3}\left(q^{2}+a_{3}\right), \quad T_{4}=-p^{2} q^{2}+q^{4}-2 a_{3}\left(p^{2}+a_{3}\right), \\
& T_{5}=p^{2} a_{2}^{2}+q^{2} a_{1}^{2}-2 a_{1} a_{2} a_{3} .
\end{aligned}
$$

Moreover, as a shorthand expedient, we will denote the arguments of several functions as a super/subscript, i.e., $f(x, y, z)=$ $f_{x y z}$ or $f(x, y, z)=f^{x y z}$. 
Then, the action of the projectors (3.8) on diagram $\left(d_{1}\right)_{\nu \mu}$ gives

$$
\begin{aligned}
A_{1}^{\left(d_{1}\right)}= & \frac{i g^{2} C_{A}}{4} \int_{\ell} \mathcal{K}^{\left(d_{1}\right)}\left\{\frac{a_{1}\left[h_{p q} \ell^{2}-T_{5}\right]}{h_{p q} \ell^{2}}\right\} \\
A_{2}^{\left(d_{1}\right)}= & -\frac{i g^{2} C_{A}}{4} \int_{\ell} \frac{\mathcal{K}^{\left(d_{1}\right)}}{h_{p q}^{2} \ell^{2}}\left\{h_{p q} \ell^{2}\left[a_{1}\left(4 a_{3}+p^{2}+3 q^{2}\right)-2 a_{2}\left(a_{3}+p^{2}\right)+2 h_{p q}\right]\right. \\
& -a_{1}\left[a_{2}^{2} h_{p q}-2 a_{2}\left(p^{2}\left(3 a_{1} a_{3}+2 a_{1} q^{2}+h_{p q}\right)+a_{3}\left(4 a_{1} a_{3}+3 a_{1} q^{2}+h_{p q}\right)\right)\right. \\
& \left.\left.+a_{1}\left(q^{2}\left(6 a_{1} a_{3}+a_{1} p^{2}+3 a_{1} q^{2}+2 h_{p q}\right)+2 a_{3}\left(a_{1} a_{3}+h_{p q}\right)\right)+3 a_{2}^{2}\left(a_{3}+p^{2}\right)^{2}\right]\right\}, \\
A_{3}^{\left(d_{1}\right)}= & \frac{i g^{2} C_{A}}{4} \int_{\ell} \frac{\mathcal{K}^{\left(d_{1}\right)}}{h_{p q}^{2} \ell^{2}}\left\{3 a_{1}^{3} q^{4}+a_{1} q^{2}\left[a_{2}\left(a_{2} p^{2}-6 a_{1} a_{3}\right)-3 h_{p q} \ell^{2}\right]+2 a_{2} a_{3}\left(a_{1} a_{2} a_{3}+h_{p q} \ell^{2}\right)\right\}, \\
A_{4}^{\left(d_{1}\right)}= & -\frac{i g^{2} C_{A}}{4} \int_{\ell} \frac{\mathcal{K}^{\left(d_{1}\right)}}{h_{p q}^{2} \ell^{2}}\left\{h_{p q} \ell^{2}\left[3 a_{1}\left(a_{3}+q^{2}\right)-2 a_{2}\left(a_{3}+p^{2}\right)+2 h_{p q}\right]\right. \\
& \left.+a_{1}\left[-a_{1} q^{2}\left(3 a_{1} a_{3}+3 a_{1} q^{2}-6 a_{2} a_{3}+2 h_{p q}\right)+2 a_{2} a_{3}\left(2 a_{1} a_{3}-a_{2} a_{3}+h_{p q}\right)-a_{2} p^{2}\left(q^{2}\left(a_{2}-2 a_{1}\right)+3 a_{2} a_{3}\right)\right]\right\}, \\
A_{5}^{\left(d_{1}\right)}= & \frac{i g^{2} C_{A}}{4} \int_{\ell} \frac{\mathcal{K}^{\left(d_{1}\right)}}{h_{p q}^{2} \ell^{2}}\left\{a _ { 1 } \left[a_{2}\left(a_{2}-2 a_{1}\right)\left(3 a_{3}^{2}+h_{p q}\right)+3 a_{1} q^{2}\left(a_{1} a_{3}+a_{1} q^{2}-2 a_{2} a_{3}\right)\right.\right. \\
& \left.\left.+3 a_{2}^{2} a_{3} p^{2}\right]-h_{p q} \ell^{2}\left[a_{3}\left(a_{1}-2 a_{2}\right)+3 a_{1} q^{2}\right]\right\},
\end{aligned}
$$

where

$$
\mathcal{K}^{\left(d_{1}\right)}=\frac{\Delta\left(\ell^{2}\right) F\left(t^{2}\right) F\left(s^{2}\right) B_{1}(s,-t, u) \mathcal{V}_{1}(\ell, q, p, r)}{s^{2} t^{2}} .
$$

Turning to diagram $\left(d_{2}\right)_{\nu \mu}$, all $A_{i}^{\left(d_{2}\right)}$ may be cast in the common form

$$
A_{i}^{\left(d_{2}\right)}=\frac{i g^{2} C_{A}}{2} \int_{\ell} \frac{\mathcal{K}_{\ell u v} S_{i}^{\ell u v}+\mathcal{K}_{u v \ell} S_{i}^{u v \ell}+\mathcal{K}_{v \ell u} S_{i}^{v \ell u}}{h_{p q}^{2} \ell^{2}},
$$

where

$$
\mathcal{K}_{x y z}=\frac{\Delta\left(\ell^{2}\right) \Delta\left(v^{2}\right) F\left(s^{2}\right) \mathcal{V}_{2}(\ell, q, p, r) X_{1}^{x y z}}{s^{2} v^{2}}
$$

Then, the $S_{i}$ are given by

$$
\begin{aligned}
S_{1}^{\ell u v}= & -h_{p q}\left\{a_{1}\left[\left(a_{3}+q^{2}\right)\left(T_{5}+h_{p q} \ell^{2}\right)-a_{2}\left(\ell^{2}\left(2 a_{3}\left(a_{3}+p^{2}\right)+h_{p q}\right)+T_{5}\right)\right]\right. \\
& \left.+a_{1}^{2}\left[a_{3} \ell^{2}\left(a_{3}+q^{2}\right)-T_{5}\right]+\left(a_{3}+p^{2}\right)\left[-a_{2} T_{5}+a_{2} \ell^{2}\left(a_{2} p^{2}-h_{p q}\right)+h_{p q} \ell^{4}\right]\right\}, \\
S_{1}^{u v \ell}= & -h_{p q}\left(a_{1}+a_{2}\right)\left(-a_{1}+a_{3}+p^{2}\right)\left[h_{p q} \ell^{2}-T_{5}\right], \\
S_{1}^{v \ell u}= & -h_{p q}\left\{T_{5}\left[a_{1}^{2}+a_{1}\left(a_{2}-a_{3}-q^{2}\right)+a_{2}\left(a_{3}+p^{2}\right)\right]+a_{1} h_{p q} \ell^{4}\right. \\
& \left.-\ell^{2}\left[a_{1}^{2}\left(h_{p q}-2 a_{2} a_{3}\right)+a_{1} q^{2}\left(a_{1}^{2}-h_{p q}\right)+a_{1} h_{p q}\left(a_{2}-a_{3}\right)+a_{2} p^{2}\left(a_{1} a_{2}+h_{p q}\right)+a_{2} a_{3} h_{p q}\right]\right\}, \\
S_{2}^{\ell u v}= & -a_{1}^{4} T_{2}+a_{1}^{3}\left[3 a_{2}\left(2 a_{3}\left(a_{3}+p^{2}\right)+p^{2} q^{2}-q^{4}\right)+\left(a_{3}+q^{2}\right)\left(3\left(a_{3}+q^{2}\right)^{2}+h_{p q}\right)\right] \\
& -a_{1}^{2}\left[3 a_{2}^{2} T_{3}+a_{2}\left(q^{2}\left(20 a_{3}^{2}+17 a_{3} p^{2}+p^{4}\right)+2 a_{3}^{2}\left(5 a_{3}+4 p^{2}\right)+q^{4}\left(9 a_{3}+7 p^{2}\right)\right)\right. \\
& -\ell^{2}\left(3 a_{3}\left(3 q^{2}\left(a_{3}+p^{2}\right)+a_{3} p^{2}+q^{4}\right)+2 h_{p q}\left(p^{2}+2 q^{2}\right)\right)+\left(\ell^{2}-a_{2}\right)\left(a_{3}+p^{2}\right)\left[a_{2}^{2} T_{1}\right. \\
& \left.-h_{p q} \ell^{2}\left(2 a_{3}+p^{2}+q^{2}\right)\right]-a_{1}\left[a_{2}^{3} T_{1}+h_{p q} \ell^{2}\left(a_{3}+q^{2}\right)\left(2 a_{3}+p^{2}+q^{2}\right)\right. \\
& -a_{2}^{2}\left(p^{2}\left(20 a_{3}^{2}+17 a_{3} q^{2}+q^{4}\right)+2 a_{3}^{2}\left(5 a_{3}+4 q^{2}\right)+p^{4}\left(9 a_{3}+7 q^{2}\right)\right) \\
& \left.+a_{2} \ell^{2}\left(p^{2}\left(15 a_{3}^{2}+8 a_{3} q^{2}-q^{4}\right)+a_{3}^{2}\left(10 a_{3}+7 q^{2}\right)+3 p^{4}\left(2 a_{3}+q^{2}\right)\right)\right],
\end{aligned}
$$




$$
\begin{aligned}
& S_{2}^{\text {uve }}=a_{1}^{2}\left[3 a_{2}^{2} T_{3}+a_{2}\left(6 a_{3} p^{4}+p^{2}\left(15 a_{3}^{2}+9 a_{3} q^{2}-h_{p q}\right)+h_{p q}\left(5 q^{2}-2 a_{3}\right)-3 a_{3} q^{2}\left(a_{3}+q^{2}\right)\right)\right. \\
& \left.-h_{p q}\left(\ell^{2}\left(4 a_{3}+p^{2}+3 q^{2}\right)+2\left(a_{3}+q^{2}\right)(p+q)^{2}\right)\right]+a_{1}^{3}\left[q^{4}\left(3 a_{2}-3 a_{3}+p^{2}\right)\right. \\
& \left.-q^{2}\left(3 p^{2}\left(a_{2}+a_{3}\right)+10 a_{3}^{2}+p^{4}\right)-2 a_{3}\left(p^{2}\left(3 a_{2}+a_{3}\right)+3 a_{3}\left(a_{2}+a_{3}\right)\right)\right]+a_{1}^{4} T_{2} \\
& +a_{1}\left[a_{2}^{3} T_{1}+a_{2}^{2}\left(p^{2}\left(14 a_{3}^{2}+5 a_{3} q^{2}+4 q^{4}\right)+2 a_{3}^{2}\left(5 a_{3}+q^{2}\right)-p^{4}\left(3 a_{3}+5 q^{2}\right)-3 p^{6}\right)\right. \\
& \left.+a_{2} h_{p q}\left(\ell^{2}\left(-2 a_{3}+p^{2}-3 q^{2}\right)+2\left(p^{2}-q^{2}\right)(p+q)^{2}\right)+h_{p q} \ell^{2}\left(a_{3}+p^{2}\right)(p+q)^{2}\right] \\
& +a_{2}\left(a_{3}+p^{2}\right)\left[(p+q)^{2}\left(h_{p q} \ell^{2}+2 a_{2} h_{p q}-3 a_{2}^{2} p^{2}\right)+2 a_{2} h_{p q}\left(\ell^{2}-a_{2}\right)\right], \\
& S_{2}^{v \ell u}=a_{1}^{4} T_{2}+a_{1}^{3}\left[3 a_{2} T_{4}-\left(a_{3}+q^{2}\right)\left(3\left(a_{3}+q^{2}\right)^{2}+h_{p q}\right)\right]+a_{1}^{2} a_{2}\left[3 a_{2} T_{3}+2 a_{3}^{2}\left(5 a_{3}+4 p^{2}\right)\right. \\
& \left.+q^{4}\left(9 a_{3}+7 p^{2}\right)\right]+\ell^{2}\left[a_{1}\left(a_{2} h_{p q}\left(-2 a_{3}+3 p^{2}-5 q^{2}\right)+3 h_{p q}\left(a_{3}+q^{2}\right)(p+q)^{2}-a_{2}^{2} T_{1}\right)\right. \\
& +a_{1}^{2}\left(p^{2}\left(6 a_{2} a_{3}+4 a_{2} q^{2}-h_{p q}\right)+q^{2}\left(6 a_{2} a_{3}-5 h_{p q}\right)+2 a_{3}\left(4 a_{2} a_{3}-3 h_{p q}\right)\right)-a_{1}^{3} T_{2} \\
& \left.+a_{2} h_{p q}\left(a_{3}+p^{2}\right)\left(4 a_{2}-3(p+q)^{2}\right)\right]+a_{1} a_{2}^{2}\left[a_{2} T_{1}-2 a_{3}^{2}\left(5 a_{3}+4 q^{2}\right)-p^{4}\left(9 a_{3}+7 q^{2}\right)\right] \\
& +a_{1} a_{2}\left[20 a_{3}^{2}\left(a_{1} q^{2}-a_{2} p^{2}\right)+17 a_{3} p^{2} q^{2}\left(a_{1}-a_{2}\right)+p^{2} q^{2}\left(p^{2} a_{1}-q^{2} a_{2}\right)\right] \\
& +h_{p q} \ell^{4}\left[a_{1}\left(4 a_{3}+p^{2}+3 q^{2}\right)-2 a_{2}\left(a_{3}+p^{2}\right)\right]+a_{2}^{3} T_{1}\left(a_{3}+p^{2}\right), \\
& S_{3}^{\ell u v}=3 a_{1}^{3} q^{6}-2 a_{2}^{2} a_{3}^{2}\left[a_{1}^{2}+a_{1} a_{2}-a_{1} a_{3}+a_{2} a_{3}+a_{2} p^{2}-\ell^{2}\left(a_{3}+p^{2}\right)\right] \\
& -a_{1} q^{4}\left[3 a_{1}\left(a_{1}^{2}+a_{1} a_{2}-a_{1} a_{3}+3 a_{2} a_{3}\right)+\ell^{2}\left(-3 a_{1} a_{3}-3 a_{1} p^{2}+h_{p q}\right)-a_{2}^{2} p^{2}\right] \\
& -q^{2}\left[-\ell^{2}\left(h_{p q}\left(a_{1}^{2}+a_{1}\left(a_{2}-a_{3}\right)+a_{2} a_{3}\right)+a_{2} p^{2}\left(-6 a_{1} a_{3}+a_{2} a_{3}+a_{2} p^{2}+h_{p q}\right)\right.\right. \\
& \left.-6 a_{1} a_{2} a_{3}^{2}\right)+h_{p q} \ell^{4}\left(a_{3}+p^{2}\right)+a_{2}\left(a_{2} p^{2}\left(a_{1}^{2}+a_{1} a_{2}-7 a_{1} a_{3}+a_{2} a_{3}+a_{2} p^{2}\right)\right. \\
& \left.\left.+a_{1}\left(a_{3}^{2}\left(9 a_{1}-8 a_{2}\right)-6 a_{1} a_{3}\left(a_{1}+a_{2}\right)+3 a_{1} h_{p q}\right)\right)\right], \\
& S_{3}^{u v \ell}=\left(a_{1}+a_{2}\right)\left[2 a_{2}\left(a_{3}^{2}\left(a_{1} a_{3}+h_{p q}\right)+q^{2}\left(a_{3}\left(a_{1}\left(a_{3}-3 a_{1}\right)+h_{p q}\right)+2 a_{1} p^{2}\left(a_{3}+q^{2}\right)\right)\right)\right. \\
& -a_{1} q^{2}\left(q^{2}\left(a_{1}\left(-3 a_{1}+3 a_{3}+p^{2}\right)+2 h_{p q}\right)+2 a_{3}\left(a_{1} a_{3}+h_{p q}\right)\right) \\
& \left.+a_{2}^{2}\left(\left(a_{1}-p^{2}\right)\left(3 a_{3}^{2}+h_{p q}\right)-3 a_{3}^{3}-5 a_{3} h_{p q}\right)\right]+2 h_{p q}^{2} \ell^{4}+\ell^{2}\left[q ^ { 2 } \left(a_{1} a_{3}^{2}\left(5 a_{1}+3 a_{2}\right)\right.\right. \\
& \left.+a_{3} h_{p q}\left(3 a_{1}+a_{2}\right)+2 h_{p q}^{2}\right)+2 a_{3}\left(-a_{2} a_{3}^{2}\left(2 a_{1}+a_{2}\right)+h_{p q}^{2}+a_{3} h_{p q}\left(a_{1}+a_{2}\right)\right) \\
& \left.+p^{2}\left(q^{2}\left(2 a_{2} a_{3}\left(2 a_{1}+a_{2}\right)+h_{p q}\left(a_{1}-3 a_{2}\right)-a_{1} q^{2}\left(5 a_{1}+3 a_{2}\right)\right)-2 a_{2} a_{3} h_{p q}\right)\right], \\
& S_{3}^{v \ell u}=-3 a_{1}^{3} q^{6}+q^{2}\left[a_{2} p^{2}\left(a_{1}^{2}\left(a_{2}+a_{3}\right)+a_{1}\left(a_{2}^{2}-6 a_{2} a_{3}-h_{p q}\right)+a_{2}^{2} a_{3}+a_{2}^{2} p^{2}\right)\right. \\
& \left.+a_{1} a_{3}\left(a_{1}^{2}\left(a_{3}-6 a_{2}\right)+a_{1}\left(-6 a_{2}^{2}+7 a_{2} a_{3}+h_{p q}\right)-2 a_{2}\left(4 a_{2} a_{3}+h_{p q}\right)\right)\right] \\
& +a_{2} a_{3}\left[a_{3}\left(a_{1}^{2}\left(2 a_{2}-a_{3}\right)+a_{1}\left(2 a_{2}^{2}-3 a_{2} a_{3}-h_{p q}\right)+a_{2}\left(2 a_{2} a_{3}+h_{p q}\right)\right)\right. \\
& \left.+a_{2} p^{2}\left(2 a_{2} a_{3}+h_{p q}\right)\right]+\ell^{2}\left[-a_{3}\left(a_{1} a_{3}\left(a_{2}\left(2 a_{2}+a_{3}\right)+h_{p q}\right)+\left(2 a_{2} a_{3}+h_{p q}\right)^{2}\right)\right. \\
& +q^{2}\left(3 a_{1} q^{2}\left(h_{p q}-a_{1}^{2}\right)+p^{2}\left(a_{1}\left(a_{2}\left(a_{3}-a_{2}\right)+h_{p q}\right)-a_{1} q^{2}\left(2 a_{1}+5 a_{2}\right)+a_{2}\left(4 a_{2} a_{3}+h_{p q}\right)\right)\right. \\
& \left.\left.+3 a_{3} h_{p q}\left(a_{1}-a_{2}\right)+a_{1} a_{3}\left(2 a_{1}\left(3 a_{2}+a_{3}\right)+5 a_{2} a_{3}\right)-h_{p q}^{2}\right)\right]+a_{1} q^{4}\left[-p^{2}\left(a_{1}-a_{2}\right)^{2}\right. \\
& \left.+a_{1}\left(-3 a_{3}\left(a_{1}-3 a_{2}\right)+3 a_{1}\left(a_{1}+a_{2}\right)+h_{p q}\right)\right]-h_{p q} \ell^{4}\left(-3 a_{1} q^{2}+2 a_{2} a_{3}+h_{p q}\right), \\
& S_{4}^{\ell u v}=-\left[a_{1}^{2}+a_{1}\left(a_{2}-a_{3}-q^{2}\right)+a_{2}\left(a_{3}+p^{2}\right)\right]\left[a_{2}^{2}\left(3 a_{3}\left(a_{3}+p^{2}\right)+h_{p q}\right)\right. \\
& \left.-2 a_{1} a_{2}\left(3 a_{3}\left(a_{3}+q^{2}\right)+h_{p q}\right)+3 a_{1}^{2} q^{2}\left(a_{3}+q^{2}\right)\right]-h_{p q} \ell^{4}\left(a_{3}+p^{2}\right)\left(a_{3}+q^{2}\right) \\
& -\ell^{2}\left[a_{1}\left(a_{3}+q^{2}\right)\left(q^{2}\left(-3 a_{1} a_{3}-4 a_{1} p^{2}+h_{p q}\right)+a_{3}\left(a_{1} a_{3}+h_{p q}\right)\right)\right. \\
& +a_{2}\left(-p^{2}\left(q^{2}\left(-7 a_{1} a_{3}+a_{1} q^{2}+h_{p q}\right)+a_{3}\left(h_{p q}-4 a_{1} a_{3}\right)\right)\right. \\
& \left.+a_{3}\left(q^{2}\left(7 a_{1} a_{3}-h_{p q}\right)+a_{3}\left(5 a_{1} a_{3}-h_{p q}\right)\right)+2 a_{1} p^{4} q^{2}\right) \\
& \left.-a_{2}^{2}\left(a_{3}+p^{2}\right)\left(3 a_{3}\left(a_{3}+p^{2}\right)+h_{p q}\right)\right] \text {, }
\end{aligned}
$$




$$
\begin{aligned}
& S_{4}^{u v \ell}=2 h_{p q}^{2} \ell^{4}+\left(a_{1}+a_{2}\right)\left[a _ { 1 } q ^ { 2 } \left(a_{3}\left(3 a_{1}^{2}-7 a_{1} a_{3}-4 h_{p q}\right)+p^{2}\left(-3 a_{1} a_{3}+a_{1} q^{2}-2 h_{p q}\right)\right.\right. \\
& \left.+q^{2}\left(3 a_{1}\left(a_{1}-a_{3}\right)-2 h_{p q}\right)\right)+a_{2}^{2}\left(2 a_{1} a_{3}^{2}+a_{1} p^{2}\left(3 a_{3}+q^{2}\right)-h_{p q}\left(2 a_{3}+p^{2}\right)\right. \\
& \left.-3 a_{3} p^{2}\left(2 a_{3}+p^{2}+q^{2}\right)\right)+2 a_{2}\left(a_{3}\left(2 a_{3}\left(-a_{1}^{2}+2 a_{1} a_{3}+h_{p q}\right)+p^{2}\left(2 a_{1} a_{3}+h_{p q}\right)\right)\right. \\
& \left.\left.+q^{2}\left(a_{1}\left(\left(a_{3}+p^{2}\right)^{2}-a_{1}\left(3 a_{3}+p^{2}\right)\right)+a_{3} h_{p q}\right)+2 a_{1} p^{2} q^{4}\right)\right] \\
& +\ell^{2}\left[q^{2}\left(-5 a_{1}^{2} h_{p q}+3 a_{1} a_{3}\left(a_{2} a_{3}+h_{p q}\right)+h_{p q}\left(a_{2} a_{3}+2 h_{p q}\right)\right)-3 a_{1} a_{2} a_{3}^{3}\right. \\
& +a_{3} h_{p q}\left(-3 a_{1}^{2}+7 a_{1} a_{3}+a_{2}\left(2 a_{2}+3 a_{3}\right)\right)-2 a_{2} p^{4}\left(h_{p q}-a_{1} q^{2}\right)+4 a_{3} h_{p q}^{2} \\
& -p^{2}\left(q^{2}\left(-3 a_{1} a_{2} a_{3}+3 a_{1} a_{2} q^{2}+a_{1} h_{p q}+3 a_{2} h_{p q}\right)+a_{1} a_{3}\left(2 a_{2} a_{3}-3 h_{p q}\right)\right. \\
& \left.\left.+h_{p q}\left(3 a_{2} a_{3}-2 h_{p q}\right)\right)\right] \text {, } \\
& S_{4}^{v \ell u}=\left[a_{1}^{2}+a_{1}\left(a_{2}-a_{3}-q^{2}\right)+a_{2}\left(a_{3}+p^{2}\right)\right]\left[3 a_{1}^{2} q^{2}\left(a_{3}+q^{2}\right)-2 a_{1} a_{2}\left(3 a_{3}\left(a_{3}+q^{2}\right)+h_{p q}\right)\right. \\
& \left.+a_{2}^{2}\left(3 a_{3}\left(a_{3}+p^{2}\right)+h_{p q}\right)\right]-\ell^{2}\left[a_{1}^{2}\left(q^{2}\left(3 h_{p q}-2 a_{2}\left(3 a_{3}+p^{2}\right)\right)+a_{3}\left(3 h_{p q}-4 a_{2} a_{3}\right)\right)\right. \\
& +a_{1}\left(a_{2}^{2}\left(3 a_{3}\left(a_{3}+p^{2}\right)+h_{p q}\right)+a_{2}\left(3 a_{3}-2 p^{2}+5 q^{2}\right) h_{p q}-3 h_{p q}\left(a_{3}+q^{2}\right)^{2}\right) \\
& \left.+3 a_{1}^{3} q^{2}\left(a_{3}+q^{2}\right)+a_{2} h_{p q}\left(a_{3}+p^{2}\right)\left(3\left(a_{3}+q^{2}\right)-4 a_{2}\right)\right] \\
& -h_{p q} \ell^{4}\left[2 a_{2}\left(a_{3}+p^{2}\right)-3 a_{1}\left(a_{3}+q^{2}\right)\right] \text {, } \\
& S_{5}^{\ell u v}=-\left[a_{1}^{2}+a_{1}\left(a_{2}-a_{3}-q^{2}\right)+a_{2}\left(a_{3}+p^{2}\right)\right]\left[-2 a_{1} a_{2}\left(3 a_{3}\left(a_{3}+q^{2}\right)+h_{p q}\right)\right. \\
& \left.+3 a_{1}^{2} q^{2}\left(a_{3}+q^{2}\right)+a_{2}^{2}\left(3 a_{3}\left(a_{3}+p^{2}\right)+h_{p q}\right)\right]-h_{p q} \ell^{4}\left(a_{3}+p^{2}\right)\left(a_{3}+q^{2}\right) \\
& -\ell^{2}\left[a _ { 2 } \left(-p^{2}\left(q^{2}\left(-7 a_{1} a_{3}+a_{1} q^{2}+h_{p q}\right)+a_{3}\left(h_{p q}-4 a_{1} a_{3}\right)\right)+2 a_{1} p^{4} q^{2}\right.\right. \\
& \left.+a_{3}\left(q^{2}\left(7 a_{1} a_{3}-h_{p q}\right)+a_{3}\left(5 a_{1} a_{3}-h_{p q}\right)\right)\right)-a_{2}^{2}\left(a_{3}+p^{2}\right)\left(3 a_{3}\left(a_{3}+p^{2}\right)+h_{p q}\right) \\
& \left.+a_{1}\left(a_{3}+q^{2}\right)\left(q^{2}\left(-3 a_{1} a_{3}-4 a_{1} p^{2}+h_{p q}\right)+a_{3}\left(a_{1} a_{3}+h_{p q}\right)\right)\right], \\
& S_{5}^{u v \ell}=\left(a_{1}+a_{2}\right)\left\{3 a_{1}^{3} q^{2}\left(a_{3}+q^{2}\right)-a_{1}^{2}\left(2 a_{2}+a_{3}+q^{2}\right)\left[2 a_{3}^{2}+q^{2}\left(3 a_{3}+p^{2}\right)\right]\right. \\
& +a_{1}\left[a_{2}^{2}\left(3 a_{3}\left(a_{3}+p^{2}\right)+h_{p q}\right)+2 a_{2}\left(a_{3}+q^{2}\right)\left(3 a_{3}\left(a_{3}+p^{2}\right)+2 h_{p q}\right)\right. \\
& \left.+\left(a_{3}+q^{2}\right)\left(-3 \ell^{2} h_{p q}-2 h_{p q}\left(a_{3}+q^{2}\right)\right)\right]-\left(a_{3}+p^{2}\right)\left[\ell ^ { 2 } \left(a_{3}\left(2 a_{2} a_{3}-h_{p q}\right)\right.\right. \\
& \left.\left.\left.-q^{2}\left(2 a_{2} p^{2}+h_{p q}\right)\right)+a_{2}\left(2 h_{p q}\left(a_{2}-a_{3}-q^{2}\right)+3 a_{2} p^{2}\left(a_{3}+q^{2}\right)\right)\right]\right\}, \\
& S_{5}^{v \ell u}=-a_{1}^{3}\left[q^{2}\left(3 q^{2}\left(-a_{2}+2 a_{3}+q^{2}\right)+3 a_{3}\left(a_{2}+a_{3}\right)+2 a_{2} p^{2}+h_{p q}\right)+a_{3}\left(4 a_{2} a_{3}+h_{p q}\right)\right] \\
& -a_{1}^{2}\left[-a_{2}\left(4 a_{3}^{3}+p^{2}\left(5 a_{3} q^{2}+h_{p q}+4 q^{4}\right)+a_{3} q^{2}\left(14 a_{3}+9 q^{2}\right)\right)-h_{p q}\left(a_{3}+q^{2}\right)^{2}\right. \\
& \left.+a_{2}^{2}\left(3 a_{3}\left(a_{3}-p^{2}+2 q^{2}\right)+h_{p q}\right)\right]-\ell^{2}\left[a _ { 3 } \left(2 a_{2} a_{3}\left(-2 a_{1}^{2}+a_{1}\left(a_{2}+a_{3}\right)+2 a_{2} a_{3}\right)\right.\right. \\
& \left.+a_{2} p^{2}\left(3 a_{1} a_{2}-a_{1} a_{3}+3 h_{p q}\right)+a_{3} h_{p q}\left(a_{1}+7 a_{2}\right)\right)+a_{1} q^{4}\left(3 a_{1}^{2}+p^{2}\left(4 a_{1}+5 a_{2}\right)-3 h_{p q}\right) \\
& +h_{p q}^{2}(p+q)^{2}-q^{2}\left(p^{2}\left(2 a_{1}^{2} a_{2}-a_{1} a_{2}\left(a_{2}+p^{2}\right)+4 a_{1} h_{p q}+a_{2} h_{p q}\right)+a_{1} a_{3}^{2}\left(4 a_{1}+5 a_{2}\right)\right. \\
& \left.\left.+a_{3}\left(-3 a_{1}^{3}+6 a_{1}^{2} a_{2}+2 a_{2} p^{2}\left(a_{1}+2 a_{2}\right)+6 a_{1} h_{p q}-3 a_{2} h_{p q}\right)\right)\right]+3 a_{1}^{4} q^{2}\left(a_{3}+q^{2}\right) \\
& -a_{1} a_{2}\left[a_{2} p^{2}\left(8 a_{3}^{2}+11 a_{3} q^{2}+q^{4}+p^{2} q^{2}\right)+a_{2} a_{3}^{2}\left(7 a_{3}+8 q^{2}\right)-a_{2}^{2}\left(3 a_{3}\left(a_{3}+p^{2}\right)+h_{p q}\right)\right. \\
& \left.+2 h_{p q}\left(a_{3}+p^{2}\right)\left(a_{3}+q^{2}\right)\right]+h_{p q} \ell^{4}\left[a_{3}\left(a_{1}-2 a_{2}+a_{3}\right)+q^{2}\left(3 a_{1}-p^{2}\right)\right] \\
& +a_{2}^{2}\left(a_{3}+p^{2}\right)\left[a_{2}\left(3 a_{3}\left(a_{3}+p^{2}\right)+h_{p q}\right)+h_{p q}\left(a_{3}+p^{2}\right)\right] \text {. }
\end{aligned}
$$

Finally, it is understood that, for the numerical evaluation of the above expressions, all relevant quantities are to be replaced by their "input" expressions, namely $\Delta_{\text {in }}(q), X_{1}^{\text {in }}(r, t, \ell), F_{\text {in }}(q)$, and $B_{1}^{\text {in }}(Q)$, introduced in Sec. IV. 
[1] C. D. Roberts and A. G. Williams, Prog. Part. Nucl. Phys. 33, 477 (1994).

[2] D. Binosi, L. Chang, J. Papavassiliou, and C. D. Roberts, Phys. Lett. B 742, 183 (2015).

[3] D. Binosi, C. Mezrag, J. Papavassiliou, C. D. Roberts, and J. Rodriguez-Quintero, Phys. Rev. D 96, 054026 (2017).

[4] D. Binosi, L. Chang, J. Papavassiliou, S.-X. Qin, and C. D. Roberts, Phys. Rev. D 93, 096010 (2016).

[5] C. S. Fischer, J. Phys. G 32, R253 (2006).

[6] C. S. Fischer, A. Maas, and J. M. Pawlowski, Ann. Phys. (Amsterdam) 324, 2408 (2009).

[7] A. C. Aguilar, D. Binosi, and J. Papavassiliou, Phys. Rev. D 78, 025010 (2008).

[8] J. Rodriguez-Quintero, J. High Energy Phys. 01 (2011) 105.

[9] P. Boucaud, J. P Leroy, A. Le Yaouanc, J. Micheli, O. Pène, and J. Rodríguez-Quintero, J. High Energy Phys. 06 (2008) 099.

[10] M. Pennington and D. Wilson, Phys. Rev. D 84, 119901 (2011).

[11] D. R. Campagnari and H. Reinhardt, Phys. Rev. D 82, 105021 (2010).

[12] R. Alkofer and L. von Smekal, Phys. Rep. 353, 281 (2001).

[13] W. Schleifenbaum, A. Maas, J. Wambach, and R. Alkofer, Phys. Rev. D 72, 014017 (2005).

[14] P. Maris, C. D. Roberts, and P. C. Tandy, Phys. Lett. B 420, 267 (1998).

[15] P. Maris and C. D. Roberts, Int. J. Mod. Phys. E 12, 297 (2003).

[16] A. C. Aguilar and A. A. Natale, J. High Energy Phys. 08 (2004) 057.

[17] K.-I. Kondo, Phys. Rev. D 74, 125003 (2006).

[18] J. Braun, H. Gies, and J. M. Pawlowski, Phys. Lett. B 684, 262 (2010).

[19] D. Binosi and J. Papavassiliou, Phys. Rev. D 77, 061702 (2008).

[20] D. Binosi and J. Papavassiliou, J. High Energy Phys. 11 (2008) 063.

[21] D. Binosi and J. Papavassiliou, Phys. Rep. 479, 1 (2009).

[22] K.-I. Kondo, Phys. Rev. D 84, 061702 (2011).

[23] P. Watson and H. Reinhardt, Phys. Rev. D 82, 125010 (2010).

[24] P. Watson and H. Reinhardt, Phys. Rev. D 85, 025014 (2012).

[25] A. C. Aguilar and J. Papavassiliou, Phys. Rev. D 83, 014013 (2011).

[26] I. C. Cloet and C. D. Roberts, Prog. Part. Nucl. Phys. 77, 1 (2014).

[27] M. Mitter, J. M. Pawlowski, and N. Strodthoff, Phys. Rev. D 91, 054035 (2015).

[28] J. Braun, L. Fister, J. M. Pawlowski, and F. Rennecke, Phys. Rev. D 94, 034016 (2016).

[29] W. Heupel, T. Goecke, and C. S. Fischer, Eur. Phys. J. A 50, 85 (2014).

[30] D. Binosi, L. Chang, J. Papavassiliou, S.-X. Qin, and C. D. Roberts, Phys. Rev. D 95, 031501 (2017).

[31] A. C. Aguilar, J.C. Cardona, M. N. Ferreira, and J. Papavassiliou, Phys. Rev. D 98, 014002 (2018).

[32] A. Blum, M. Q. Huber, M. Mitter, and L. von Smekal, Phys. Rev. D 89, 061703 (2014).
[33] G. Eichmann, R. Williams, R. Alkofer, and M. Vujinovic, Phys. Rev. D 89, 105014 (2014).

[34] A. C. Aguilar, D. Binosi, D. Ibañez, and J. Papavassiliou, Phys. Rev. D 89, 085008 (2014).

[35] M. Pelaez, M. Tissier, and N. Wschebor, Phys. Rev. D 88, 125003 (2013).

[36] F. Siringo, Phys. Rev. D 94, 114036 (2016).

[37] F. Siringo and G. Comitini, Phys. Rev. D 98, 034023 (2018).

[38] R. Bermudez, L. Albino, L. X. Gutirrez-Guerrero, M. E. Tejeda-Yeomans, and A. Bashir, Phys. Rev. D 95, 034041 (2017).

[39] A. C. Aguilar, D. Binosi, D. Ibañez, and J. Papavassiliou, Phys. Rev. D 90, 065027 (2014).

[40] A. C. Aguilar, D. Binosi, and J. Papavassiliou, Phys. Rev. D 91, 085014 (2015).

[41] A. C. Aguilar, J.C. Cardona, M. N. Ferreira, and J. Papavassiliou, Phys. Rev. D 96, 014029 (2017).

[42] J. M. Cornwall and J. Papavassiliou, Phys. Rev. D 40, 3474 (1989).

[43] J. M. Cornwall, Phys. Rev. D 26, 1453 (1982).

[44] A. C. Aguilar, A. Mihara, and A. A. Natale, Phys. Rev. D 65, 054011 (2002).

[45] A. C. Aguilar, A. A. Natale, and P. S. R. da Silva, Phys. Rev. Lett. 90, 152001 (2003).

[46] N. Vandersickel and D. Zwanziger, Phys. Rep. 520, 175 (2012).

[47] D. Dudal, O. Oliveira, and J. Rodriguez-Quintero, Phys. Rev. D 86, 105005 (2012).

[48] P. Boucaud, D. Dudal, J. Leroy, O. Pene, and J. RodriguezQuintero, J. High Energy Phys. 12 (2011) 018.

[49] M. Q. Huber and L. von Smekal, J. High Energy Phys. 04 (2013) 149.

[50] B. W. Mintz, L. F. Palhares, S. P. Sorella, and A. D. Pereira, Phys. Rev. D 97, 034020 (2018).

[51] A. Cucchieri and T. Mendes, Proc. Sci. LAT2007 (2007) 297.

[52] A. Cucchieri and T. Mendes, Proc. Sci. QCD-TNT09 (2009) 026.

[53] A. Cucchieri and T. Mendes, Phys. Rev. Lett. 100, 241601 (2008).

[54] I. L. Bogolubsky, E. M. Ilgenfritz, M. Muller-Preussker, and A. Sternbeck, Proc. Sci. LATTICE2007 (2007) 290.

[55] I. Bogolubsky, E. Ilgenfritz, M. Muller-Preussker, and A. Sternbeck, Phys. Lett. B 676, 69 (2009).

[56] O. Oliveira and P. Silva, Proc. Sci. LAT2009 (2009) 226.

[57] O. Oliveira, P. J. Silva, J.-I. Skullerud, and A. Sternbeck, arXiv:1809.02541.

[58] O. Oliveira and P. Bicudo, J. Phys. G 38, 045003 (2011).

[59] O. Oliveira and P. J. Silva, Phys. Rev. D 86, 114513 (2012).

[60] P. O. Bowman, U. M. Heller, D. B. Leinweber, M. B. Parappilly, A. G. Williams, and J.-b. Zhang, Phys. Rev. D 71, 054507 (2005).

[61] A. Ayala, A. Bashir, D. Binosi, M. Cristoforetti, and J. Rodriguez-Quintero, Phys. Rev. D 86, 074512 (2012).

[62] A. Sternbeck, P.-H. Balduf, A. Kizilersu, O. Oliveira, P. J. Silva, J.-I. Skullerud, and A. G. Williams, Proc. Sci. LATTICE2016 (2017) 349.

[63] E.-M. Ilgenfritz, M. Muller-Preussker, A. Sternbeck, A. Schiller, and I. Bogolubsky, Braz. J. Phys. 37, 193 (2007). 
[64] A. Sternbeck, The infrared behavior of lattice QCD Green's functions, Ph.D. thesis, Humboldt-University Berlin, 2006.

[65] A. Kizilersu, D. B. Leinweber, J.-I. Skullerud, and A. G. Williams, Eur. Phys. J. C 50, 871 (2007).

[66] J. I. Skullerud, P. O. Bowman, A. Kizilersu, D. B. Leinweber, and A. G. Williams, J. High Energy Phys. 04 (2003) 047.

[67] P. Boucaud, F. De Soto, J. Rodrguez-Quintero, and S. Zafeiropoulos, Phys. Rev. D 95, 114503 (2017).

[68] A. Athenodorou, D. Binosi, P. Boucaud, F. De Soto, J. Papavassiliou, J. Rodriguez-Quintero, and S. Zafeiropoulos, Phys. Lett. B 761, 444 (2016).

[69] A. Cucchieri, A. Maas, and T. Mendes, Phys. Rev. D 77, 094510 (2008).

[70] A. Cucchieri, A. Maas, and T. Mendes, Phys. Rev. D 74, 014503 (2006).

[71] A. C. Aguilar, D. Ibañez, and J. Papavassiliou, Phys. Rev. D 87, 114020 (2013).

[72] M. Q. Huber, A. Maas, and L. von Smekal, J. High Energy Phys. 11 (2012) 035.

[73] A. Maas, Phys. Rep. 524, 203 (2013).

[74] R. Williams, C. S. Fischer, and W. Heupel, Phys. Rev. D 93, 034026 (2016).

[75] G. Eichmann, C. S. Fischer, and W. Heupel, Phys. Rev. D 92, 056006 (2015).

[76] G. Eichmann, I. C. Cloet, R. Alkofer, A. Krassnigg, and C. D. Roberts, Phys. Rev. C 79, 012202 (2009).

[77] L. Corell, A. K. Cyrol, M. Mitter, J. M. Pawlowski, and N. Strodthoff, SciPost Phys. 5, 066 (2018).

[78] A. K. Cyrol, M. Mitter, J. M. Pawlowski, and N. Strodthoff, Phys. Rev. D 97, 054006 (2018).

[79] A. K. Cyrol, L. Fister, M. Mitter, J. M. Pawlowski, and N. Strodthoff, Phys. Rev. D 94, 054005 (2016).

[80] E. Rojas, J. de Melo, B. El-Bennich, O. Oliveira, and T. Frederico, J. High Energy Phys. 10 (2013) 193.

[81] O. Oliveira, T. Frederico, W. de Paula, and J.P. B. C. de Melo, Eur. Phys. J. C 78, 553 (2018).

[82] A. Salam, Phys. Rev. 130, 1287 (1963).

[83] A. Salam and R. Delbourgo, Phys. Rev. 135, B1398 (1964).

[84] R. Delbourgo and P. C. West, J. Phys. A 10, 1049 (1977).

[85] R. Delbourgo and P. C. West, Phys. Lett. 72B, 96 (1977).
[86] W. J. Marciano and H. Pagels, Phys. Rep. 36, 137 (1978).

[87] J. S. Ball and T.-W. Chiu, Phys. Rev. D 22, 2550 (1980).

[88] A. C. Aguilar, D. Binosi, J. Papavassiliou, and J. RodriguezQuintero, Phys. Rev. D 80, 085018 (2009).

[89] D. Binosi and J. Papavassiliou, J. High Energy Phys. 03 (2011) 121.

[90] A. C. Aguilar, D. Binosi, and J. Papavassiliou, Phys. Rev. D 84, 085026 (2011).

[91] D. Binosi, D. Ibañez, and J. Papavassiliou, Phys. Rev. D 86, 085033 (2012).

[92] A. C. Aguilar, D. Binosi, and J. Papavassiliou, Phys. Rev. D 89, 085032 (2014).

[93] A. C. Aguilar, D. Binosi, C. T. Figueiredo, and J. Papavassiliou, Eur. Phys. J. C 78, 181 (2018).

[94] A. I. Davydychev, P. Osland, and O. V. Tarasov, Phys. Rev. D 54, 4087 (1996).

[95] A. C. Aguilar, D. Binosi, and J. Papavassiliou, Front. Phys. (Beijing) 11, 111203 (2016).

[96] D. Ibañez and J. Papavassiliou, Phys. Rev. D 87, 034008 (2013).

[97] L. von Smekal, A. Hauck, and R. Alkofer, Ann. Phys. (N.Y.) 267, 1 (1998); 269, 182(E) (1998).

[98] P. Watson, arXiv:hep-ph/9901454.

[99] M. Q. Huber, Ph.D. thesis, Graz University, 2018.

[100] M. Q. Huber, Eur. Phys. J. C 77, 733 (2017).

[101] J. C. Taylor, Nucl. Phys. B33, 436 (1971).

[102] A. K. Cyrol, M. Q. Huber, and L. von Smekal, Eur. Phys. J. C 75, 102 (2015).

[103] H. H. Patel, Comput. Phys. Commun. 197, 276 (2015).

[104] H. H. Patel, Comput. Phys. Commun. 218, 66 (2017).

[105] A. C. Aguilar and J. Papavassiliou, Eur. Phys. J. A 35, 189 (2008).

[106] P. Boucaud, F. De Soto, J. Leroy, A. Le Yaouanc, J. Micheli, O. Pène, and J. Rodríguez-Quintero, Phys. Rev. D 79, 014508 (2009).

[107] J. Berntsen, T. O. Espelid, and A. Genz, ACM Trans. Math. Softw. 17, 452 (1991).

[108] D. Binosi and J. Papavassiliou, Phys. Rev. D 97, 054029 (2018).

[109] S.-S. Xu, Z.-F. Cui, L. Chang, J. Papavassiliou, C. D. Roberts, and H.-S. Zong, arXiv:1805.06430.

[110] W. Celmaster and R. J. Gonsalves, Phys. Rev. D 20, 1420 (1979). 